\title{
Environmental Superstatistics
}

\author{
G.Cigdem Yalcin ${ }^{1 *}$ and Christian Beck $^{2}$ \\ ${ }^{1}$ Department of Physics, Istanbul University, 34134, Vezneciler, Istanbul, Turkey \\ ${ }^{2}$ School of Mathematical Sciences, Queen Mary, University of London, London E1 4NS, UK
}

\begin{abstract}
A thermodynamic device placed outdoors, or a local ecosystem, is subject to a variety of different temperatures given by short-term (daily) and long-term (seasonal) variations. In the long term a superstatistical description makes sense, with a suitable distribution function $f(\beta)$ of inverse temperature $\beta$ over which ordinary statistical mechanics is averaged. We show that $f(\beta)$ is very different at different geographic locations, and typically exhibits a double-peak structure for longterm data. For some of our data sets we also find a systematic drift due to global warming. For a simple superstatistical model system we show that the response to global warming is stronger if temperature fluctuations are taken into account.
\end{abstract}

\section{INTRODUCTION}

In nonequilibrium statistical mechanics, the superstatistics technique [1] is a powerful tool to describe a large variety of complex systems for which there is a change of environmental conditions and temperature fluctuations on a large scale [2 10]. A superstatistical complex system is mathematically described as a superposition of several statistics, one corresponding to local equilibrium statistical mechanics and the other one corresponding to a slowly varying parameter $\beta$ of the system. Essential for this approach is the fact that there is sufficient time scale separation, i.e. the local relaxation time of the system must be much shorter than the typical time scale on which $\beta$ changes. Many interesting applications of the superstatistics concept have been worked out for a variety of complex systems, for example the analysis of train delay statistics [11], hydrodynamic turbulence [12], cancer survival statistics [13] and some other applications as well, see [14 19].

In this paper we want to deal with environmental aspects of superstatistics, in the sense that we ask what distributions of inverse temperatures are seen by thermodynamic devices that are kept in open air outside a constanttemperature environment. Clearly this question is technically very relevant as many devices need to operate under strong temperature fluctuations, as given by either daily temperature fluctuations, or seasonal variations, or even long-term climatic changes. Besides thermodynamic devices, one may also be interested in local complex ecosystems (such as e.g. biological populations) coupled to a changing temperature environment. We will analyse in detail inverse temperature distributions at various geographic locations. These environmentally important distributions are different from standard examples of distribution functions discussed so far in the literature, such as the $\chi$-square, inverse $\chi$-square or lognormal distribution [2]. As it is well-known, superstatistics based on $\chi$-square distributions leads to $q$-statistics [20, 21], whereas other distributions lead to something more complicated. A major difference is that the environmentally observed probability densities of inverse temperature typically exhibit a double-peak structure, thus requiring a different type of superstatistics than what has been done so far. In the original setting described in [1], the analysis was based on single-peaked distributions with a sharp maximum [1]. For environmentally relevant temperature distributions, this concept has to be broadened.

A central point for the applicability of the superstatistics concept is the existence of suitable time scale separation of the dynamical evolution, or more generally the existence of a hierarchy of time scales which are well separated. In the simplest case this just means there are two different time scales such that the typical variation of $\beta$ takes place on a much larger time scale than the local relaxation time of the system that is influenced by the given temperature environment. For meteorological and climatic systems there is indeed a hierarchy of different relevant time scales. It starts with time scales due to the turbulent dynamics of the air which are well below the daily temperature oscillations; also there are synoptic meteorological disturbances of the order of about 2 days. We arrive at seasonal variations (circular statistics) and at larger scales at inter-annual variability. Finally, at largest scales there are long-term trends due to climatic and geological changes. Our aim in this paper is not a detailed analysis of all these various dynamical phenomena on various time scales (see, e.g. [41 45] and references therein), but the application of superstatistical techniques, given the fact that there is time scale separation. This means effectively we consider a given subsystem or device (which may be a technical thermodynamic device, but in a more general setting also a given local ecosystem depending on temperature and precipitation dynamics) and then look at a generalized statistical mechanics description of it. For this it is necessary to fully understand the statistics of (inverse) temperature fluctuations - either as sampled

* corresponding author, phone +90 2124555700 ext 15489, Fax +90 212455 5855, gcyalcin@istanbul.edu.tr 
over long-term records or conditioned on particular periods (say summer or winter). While in principle one could also do superstatistics on shorter (turbulent) time scales of air movement, in this paper we restrict ourselves to the long-term statistical properties.

This paper is organized as follows. In section 2 we look at monthly data (essentially eliminating seasonal variations) at various geographical locations, and check how well the data are described by Gaussian distributions. In section 3 we look at long-term data including seasonal variations, which induce double-peaked distributions, but with specific differences at different geographical locations, depending on local climate. In section 4 the results are interpreted in terms of the Köppen-Geiger climate classification scheme. In section 5 we discuss why the superstatistics relevant for environmental temperature fluctuations is different from what has been done so far for single-peaked distributions. In section 6 we deal with global warming, and the interesting effects that occur due to fluctuations in superstatistical models if a parameter such as the mean of the temperature distributions increases slightly. Finally, our conclusions are summarized in section 7 .

\section{OBSERVED INVERSE TEMPERATURE DISTRIBUTIONS-MONTHLY DATA}

When looking of temperature distributions as given by real data, one clearly has to specify the relevant time scale first. Short-term temperature distributions (dominated by daily fluctuations) are very different from long-term data (dominated by seasonal variations). For very long data records, one also has to take into account non-stationary behaviour due to climate change.

We start with short-term data. Fig. 1, 2 and 3 show as an example a time series of hourly measured surface inverse temperatures in Ottawa during November 2011, as well as in Vancouver during May 2011 and December 2011.

The frequency pattern is dominated by 1 oscillation per $24 \mathrm{~h}$, corresponding to the day-night temperature differences. Superimposed to this are stochastic fluctuations due to different weather conditions and turbulent fluctuations. What is also clearly visible in the figures are small systematic trends. In Fig. 1 (November in Ottawa) the average inverse temperature $\beta$ is slightly increasing over the month of November, since the temperature slightly decreases as winter is approaching. In Fig. 2 (May in Vancouver) the average $\beta$ is slightly decreasing, since summer is approaching. No clear systematic trend is visible for the December data of Fig. 3. 


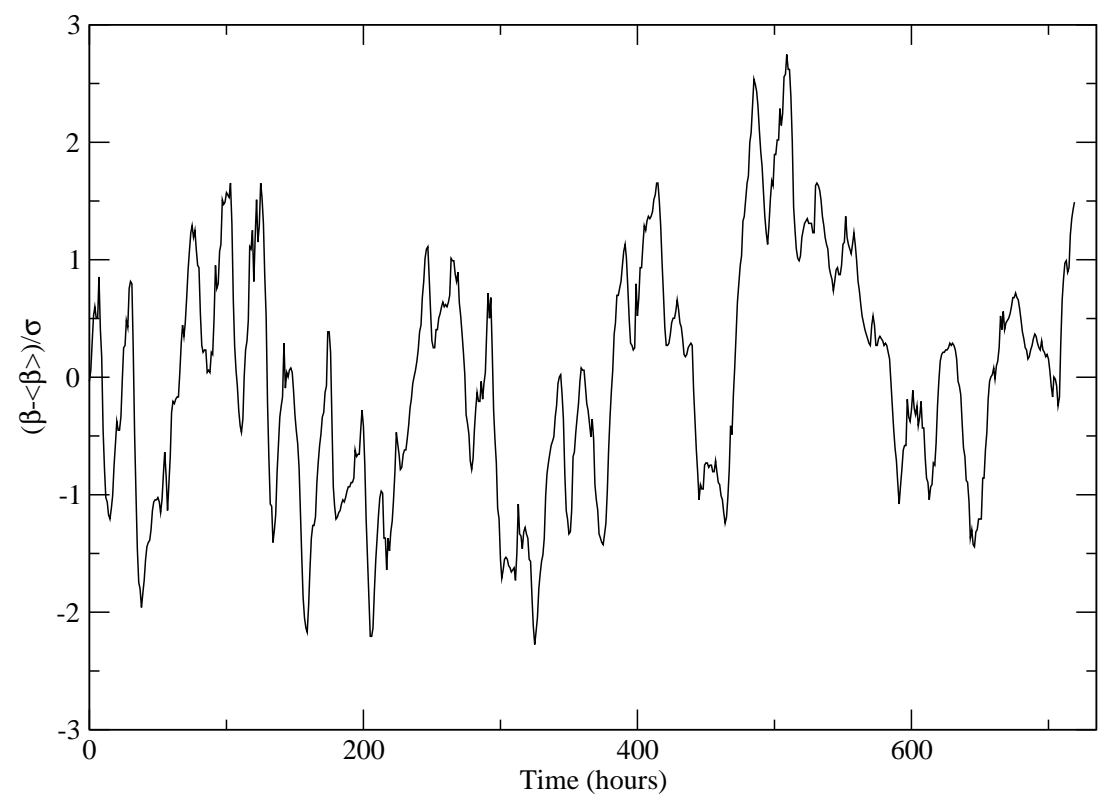

FIG. 1: Time series of hourly measured inverse temperature in Ottawa during November 2011

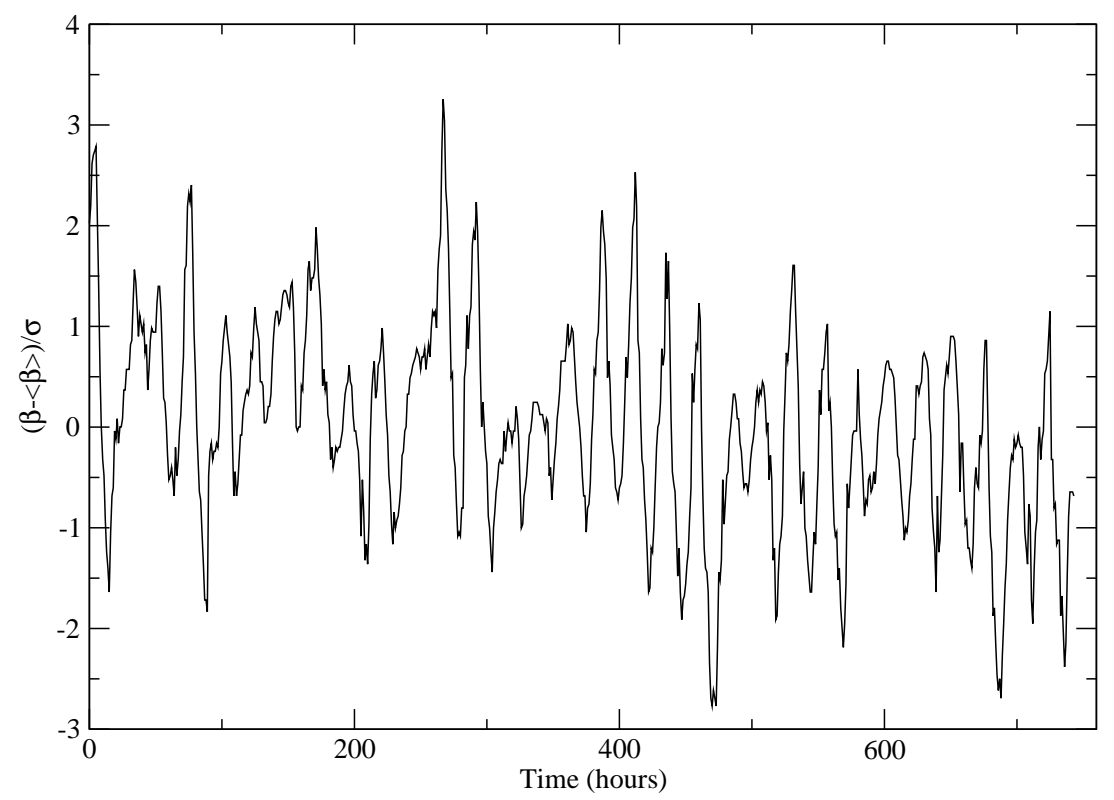

FIG. 2: Time series of hourly measured inverse temperature in Vancouver during May 2011 


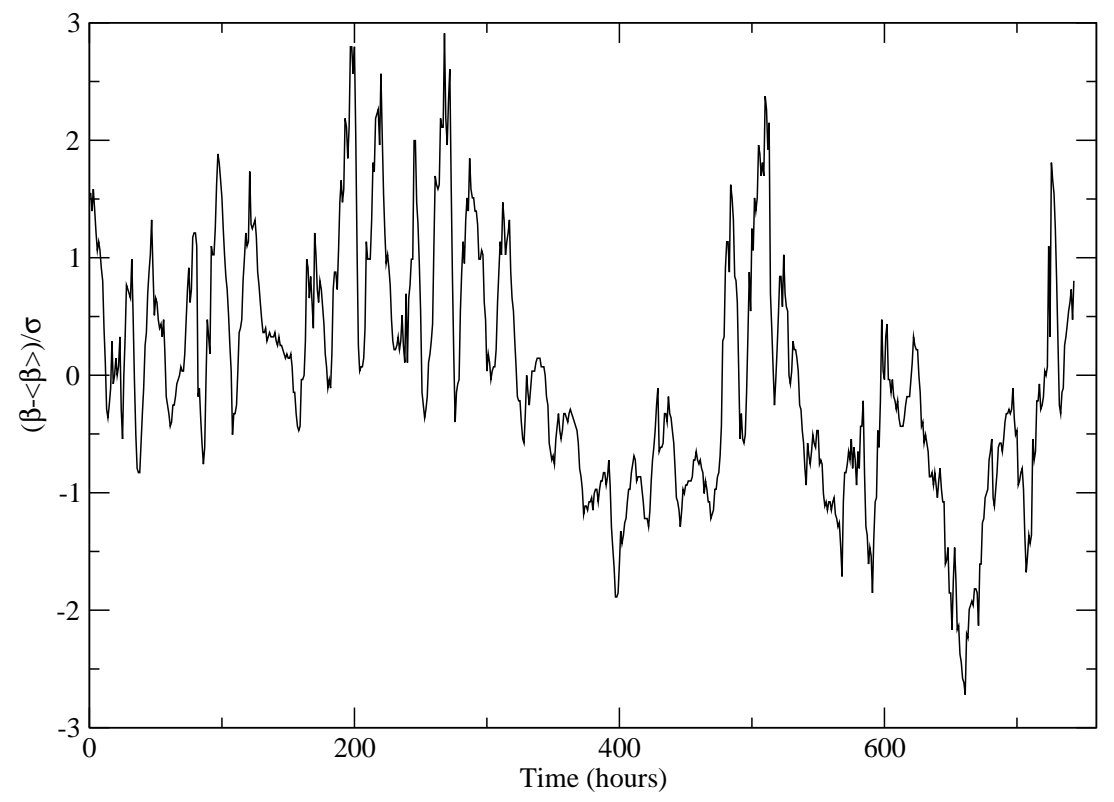

FIG. 3: Time series of hourly measured inverse temperature in Vancouver during December 2011

The (inverse) temperature time series for a single month is approximately Gaussian distributed. This is illustrated in Figs. 4-6, which shows the histograms of the example time series of Figs 1-3.

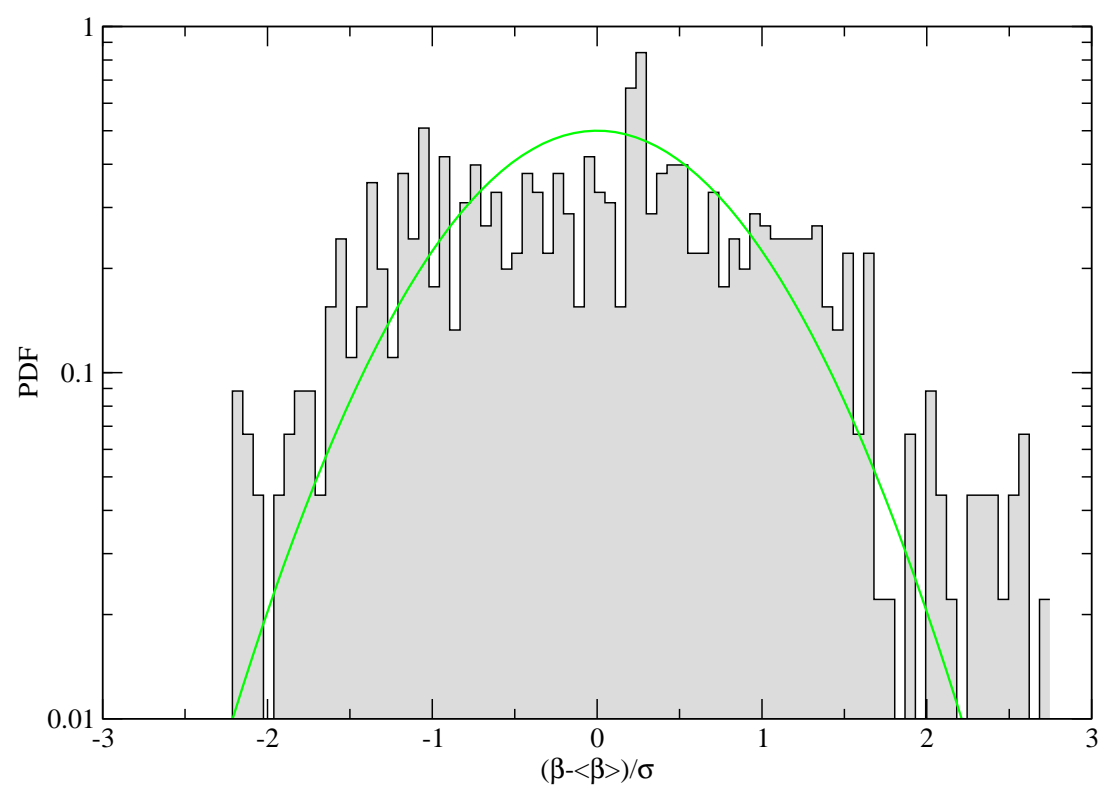

FIG. 4: Gaussian fit to the histogram of the hourly measured inverse temperature in Ottawa during November 2011 


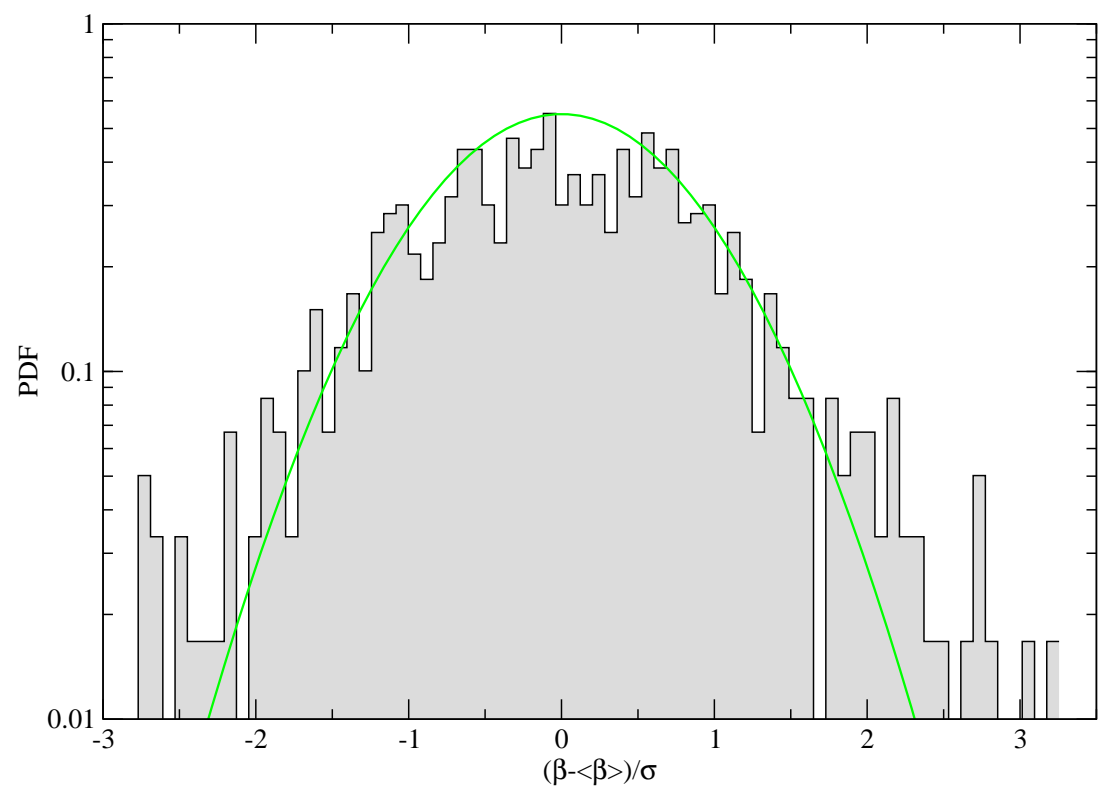

FIG. 5: Gaussian fit to the histogram of the hourly measured inverse temperature in Vancouver during May 2011

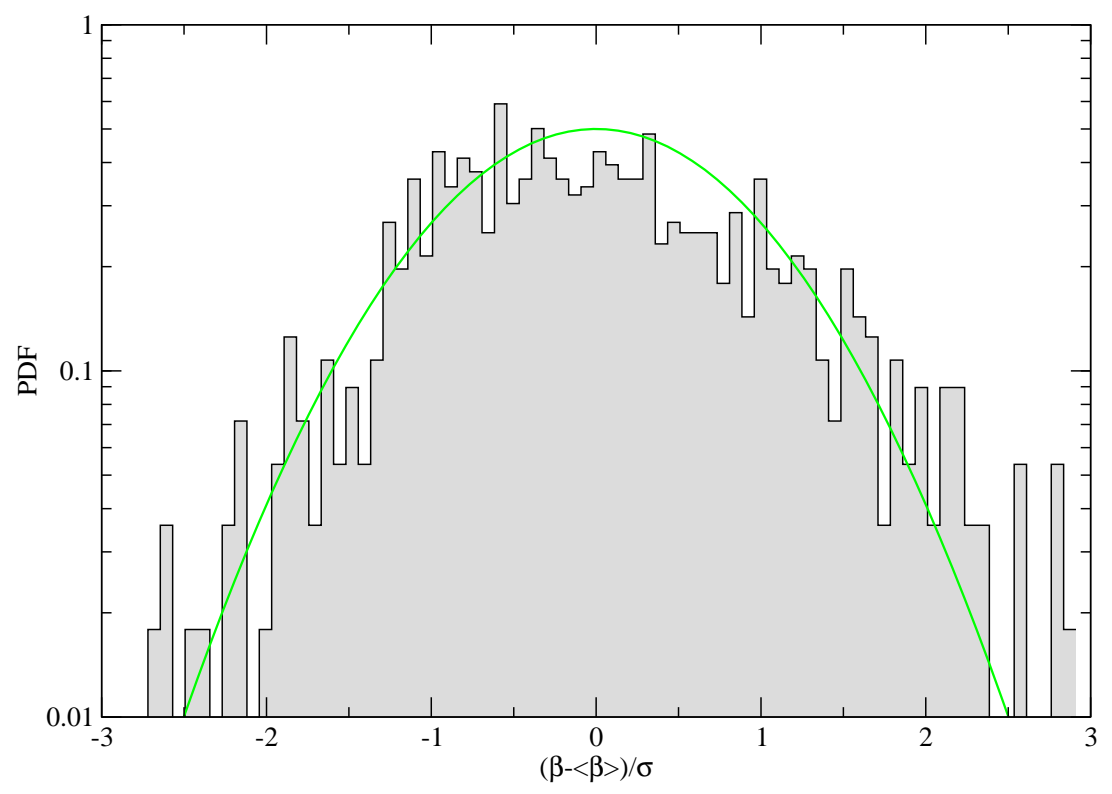

FIG. 6: Gaussian fit to the histogram of the hourly measured inverse temperature in Vancouver during December 2011

Consider now a thermodynamic device with energy levels $E_{i}$ that is kept outside and hence subject to slowly varying inverse temperatures. Our interpretation of 'thermodynamic device' is very general here. One may also think of an ecosystem or other complex system (including biological populations) that is influenced or in fact, dependent, on the temperature (and precipitation) of its environment. Suppose that locally, for constant inverse temperature, the device is properly described by statistical mechanics with ordinary Boltzmann factors $e^{-\beta E}$. Assuming time scale separation, so that the system can quickly enough relax to local equilibrium, the long-term behaviour is properly described by a mixture of Boltzmann factors with different temperatures, weighted with a function $f(\beta)$ that describes how often 
a given inverse temperature of the environment is observed. This is the realm of superstatistics [1]. The effective statistical mechanics of this thermodynamic device (or ecosystem) is indeed described by effective Boltzmann factors $B(E)$ of the form

$$
B(E)=\int_{0}^{\infty} f(\beta) e^{-\beta E} d \beta
$$

In our case, for monthly distributions as displayed in Fig. 4-6, $f(\beta)$ is indeed a single-humped probability density around the mean inverse temperature that month, in good approximation given by a Gaussian distribution. The integration over $\beta$ yields non-Boltzmannian distributions $B(E)$, whose details depend on $f(\beta)$. So for example the marginal distribution of velocities of an ideal gas for which $\beta$ fluctuates is not Gaussian anymore, due to the integration over $\beta$ in eq. (1).

Better statistics is obtained if we sample the data of a given month over many years. We sampled hourly measured inverse temperature time series, restricted to the month of November, over the period 1966-2011 for Ottawa. Fig. 7 shows this time series (restricted to November months and joined together). The corresponding histogram is well-fitted by a Gaussian (Fig. 8).

However, let us remark that Gaussian distributions in (inverse) temperature can of course only be an approximation. They cannot be exact since in principle they allow for negative $\beta$, whereas $\beta$ should always be positive. A better model would, for example, be a $\chi$-square distribution in $\beta$, or a lognormal distribution, which always has positive $\beta$. Close to their maxima these distributions still look similar to Gaussians. We should remark at this point that temperature distributions at various locations on earth have of course been analysed before. A recent paper [46] provides evidence that the distributions (when seasonal fluctuations are eliminated) are sometimes Gaussian, sometimes non-Gaussian, depending on location. While for our data in typical cases we see near-Gaussian behavior, in [46] evidence for power-law distributions and exponential tails at some special locations has been provided.

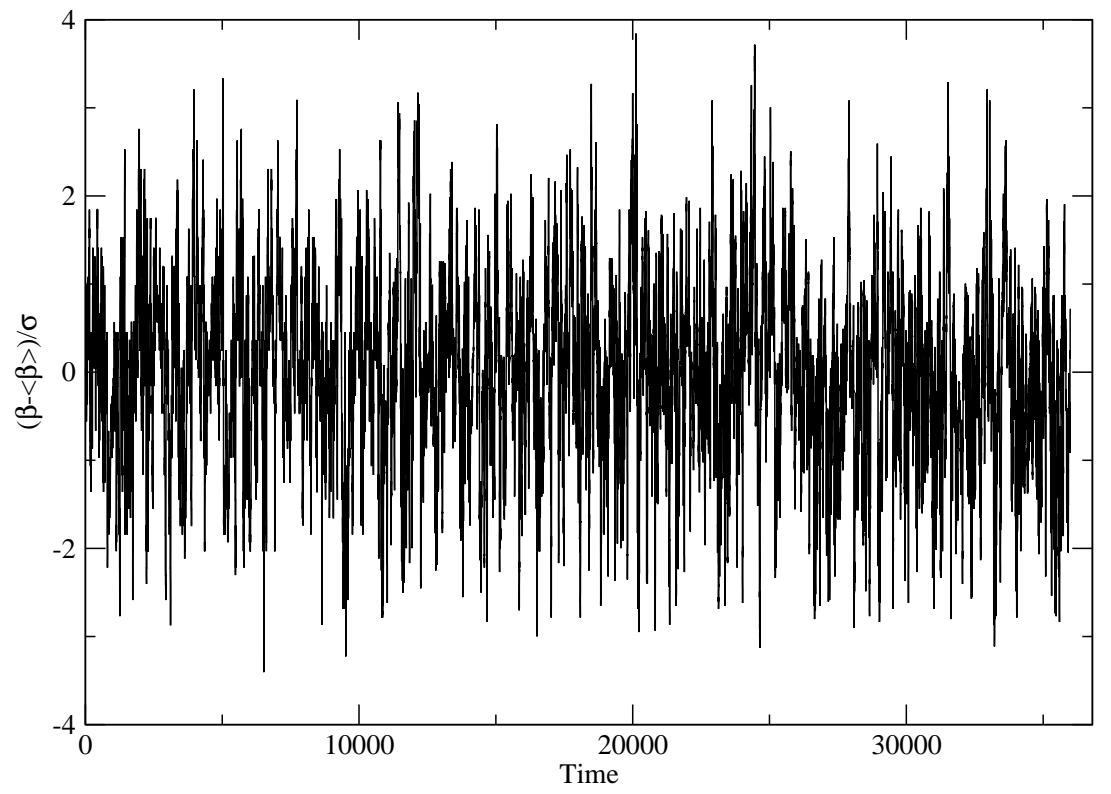

FIG. 7: Time series of hourly measured inverse temperature in Ottawa for November, sampled from 1962 to 2011 for 50 years 


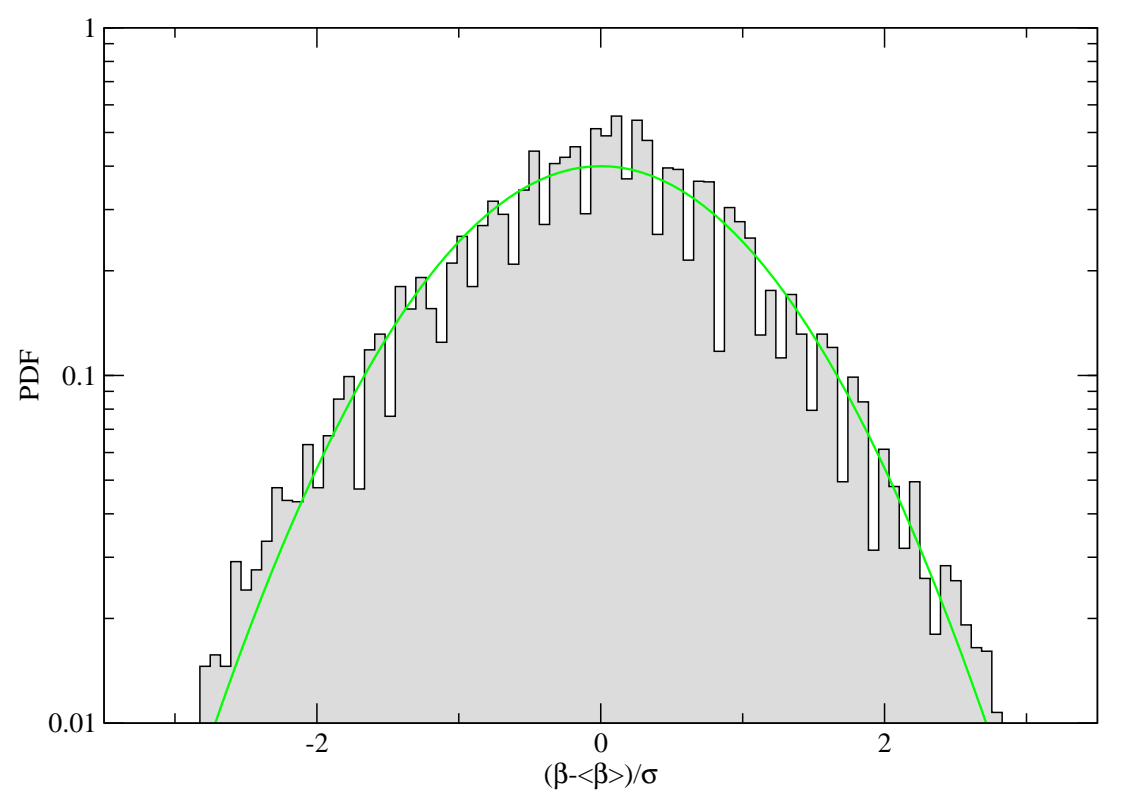

FIG. 8: Gaussian fit to the histogram of the hourly measured inverse temperature in Ottawa for 50 November months

What is generally interesting in our data is that there is a systematic trend of a changing mean on long time scales. Close inspection of Fig. 7 shows already to the bare eye that the average $\beta$ is slightly decreasing over the years, meaning the average November temperature has slightly increased in Ottawa over the past 50 years. Clearly, this effect is related to global warming, here measured at a particular location. The same trend of a slightly decreasing average $\beta$ is also visible for the May-data of Vancouver (Fig. 9), which overall are also well fitted by a Gaussian distribution (Fig. 10). Slightly more volatile behaviour is observed in December (Fig. 11), with some outbursts to large $\beta$ (some very cold days) occurring relatively frequently, see Fig. 12.

In line with the observations in [46], we see for particular seasons and particular locations deviations from Gaussian behavior, although this seems somewhat exceptional. For example, Figure 12 (winter in Vancouver) is an example where there seems to be an exponential tail for large values of $\beta$. 


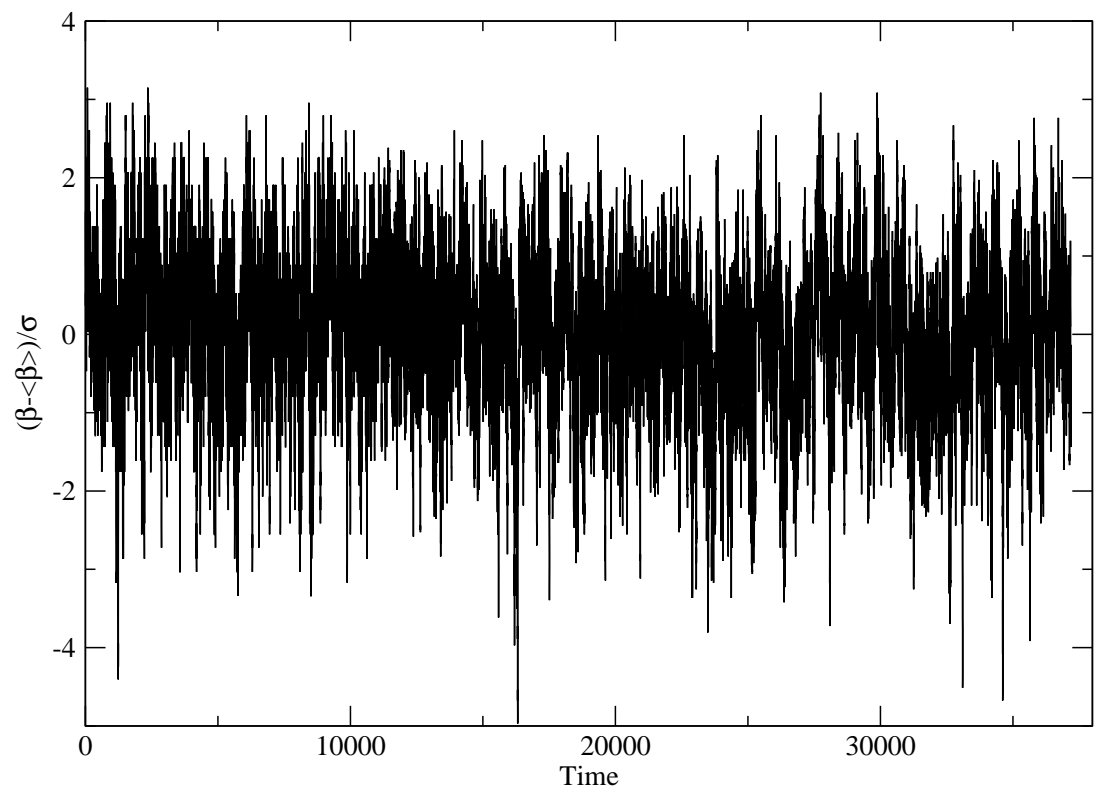

FIG. 9: Time series of hourly measured inverse temperature in Vancouver for 50 months of May

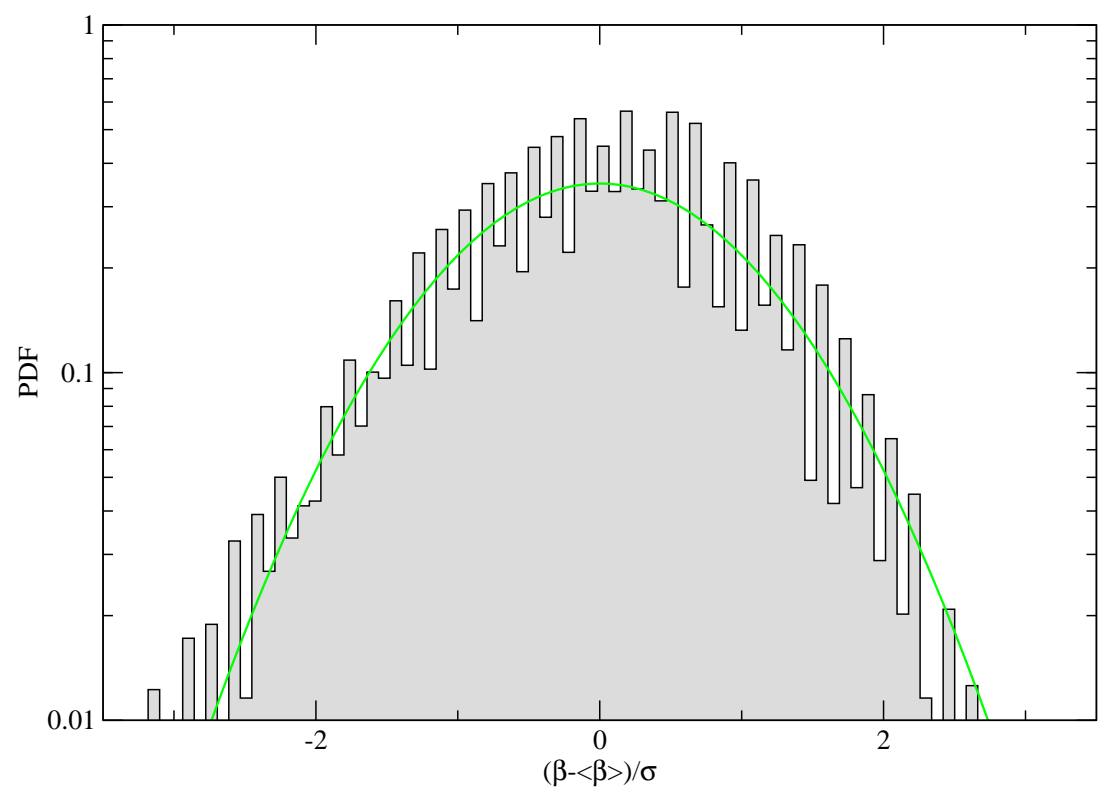

FIG. 10: Histogram the hourly measured inverse temperatures in Vancouver for 50 months of May 


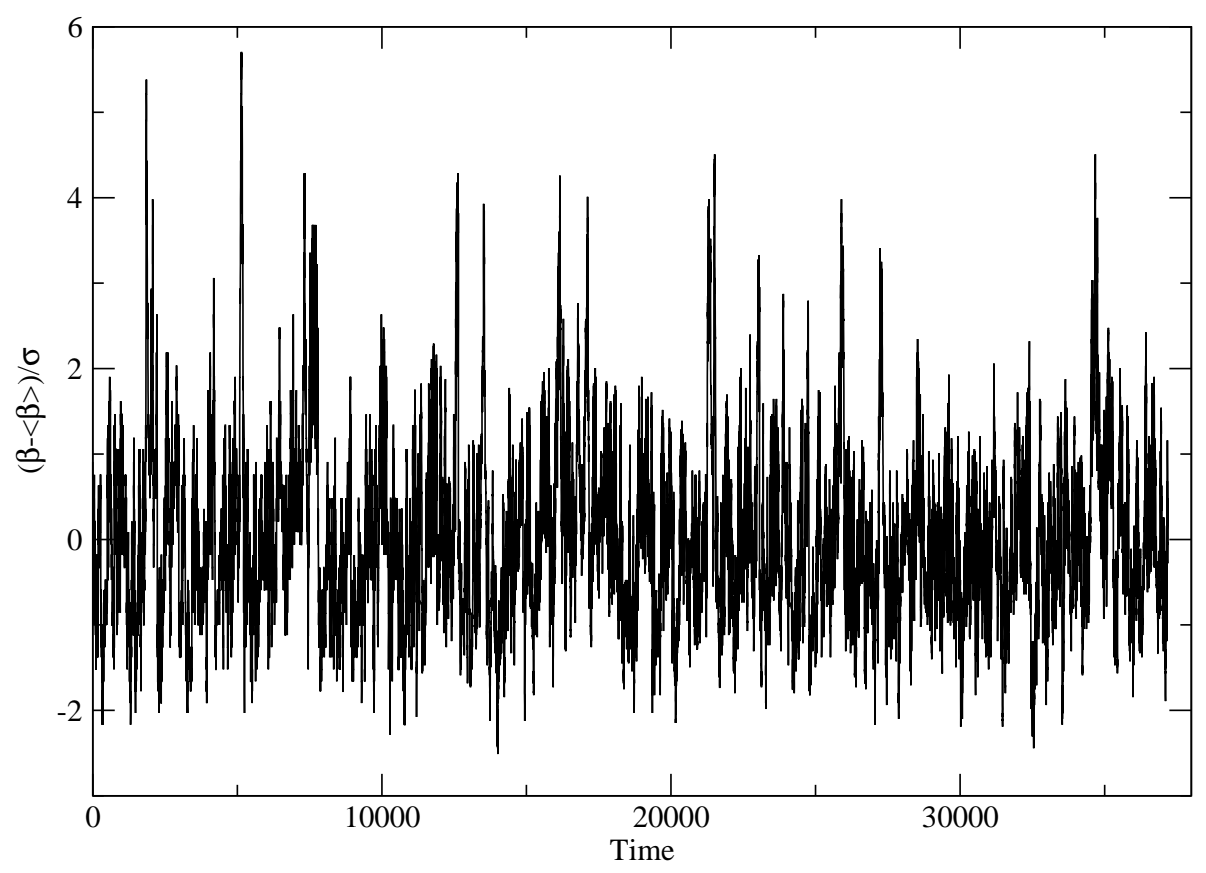

FIG. 11: Same as Fig. 9 but for December

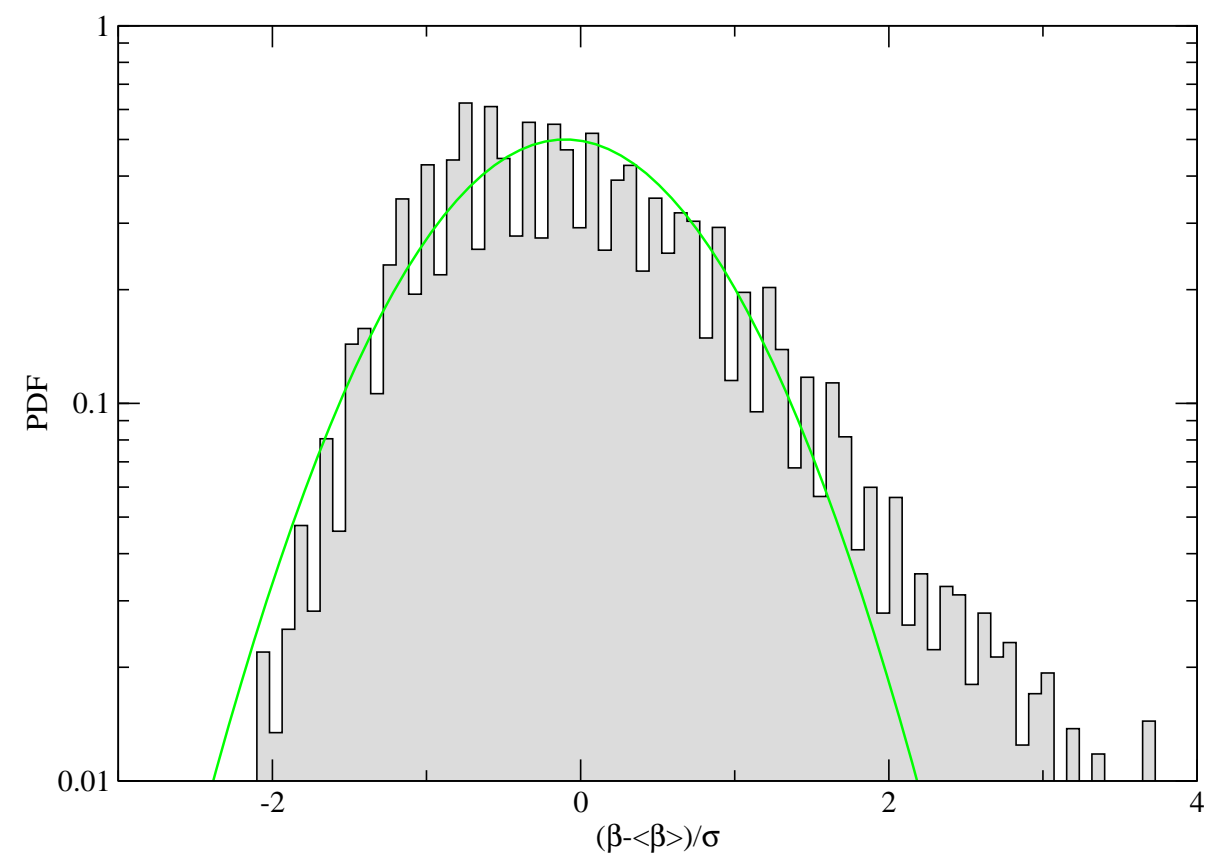

FIG. 12: Same as Fig. 10 but for December 
Typically, the distributions for most months are in reasonably good approximation Gaussian distributions. They describe random-like fluctuations around the mean temperature in that month with oscillating behaviour superimposed due to day-night temperature differences. In addition, on very long time scales there is a small systematic drift of the mean temperature to larger values, due to climate change. A thermodynamic device, to be used only in a particular month of the year, would be typically described by a superstatistics where the relevant inverse temperature distribution $f(\beta)$ is close to a Gaussian, at least close to its maximum, and where the mean of that Gaussian (and possibly also the variance) has a very small time dependence over a time scale of decades.

\section{OBSERVED INVERSE TEMPERATURE DISTRIBUTIONS - YEARLY DATA}

We now deal with thermodynamic devices (or complex local ecosystems) that are kept outside during the entire year, so that full seasonal variations are becoming relevant. The observed probability distributions become more complex, depending on the climatic conditions at the particular location. Examples of time series that we investigated in somewhat more detail are shown in Figs. 13-21, these are daily measured inverse mean temperatures in various locations sampled over many years. Clearly, the day-night oscillations are now less relevant, and what becomes dominating are seasonal variations 23 25]. The data now have a dominating frequency corresponding to 1 oscillation per 365 days due to seasonal variations, modulated by stochastic fluctuations due to different weather conditions on a time scale of days, plus large-scale annual fluctuations (some years warmer or colder than others) and systematic trends due to climate change.

We have investigated time series data for 8 different locations in different climatic zones. These are Darwin (Northern Territory, Australia) (1975-2011) [28], Santa Fe (New Mexico, USA) (1998-2011) 29], Dubai (United Arab Emirates)(1974-2011) 30], Sydney (New South Wales, Australia) (1910-2011) 28], Central England (London, Bristol, Lancashire) (1910-2011) 31, 32], Vancouver (British Columbia, Canada) (1937-2011) 33], Hong Kong (PRC) (19972011) [34], Ottawa (Ontario, Canada) (1939-2011) [33], and Eureka (Nunavut, Canada) (1951-2011) [33], respectively.

These time series are heavily influenced by seasonal variations and fluctuations around the typical yearly behaviour. Interesting enough, for some locations a systematic trend (a decrease in the average inverse temperature over the years) is again visible to the bare eye. The locations where such a systematic trend is clearly seen are Dubai (Fig. 15), Sydney (Fig. 16), Vancouver (Fig. 18), Ottawa (Fig. 20), and Eureka (Fig. 21). Obviously these are local manifestations of the effects of global warming.

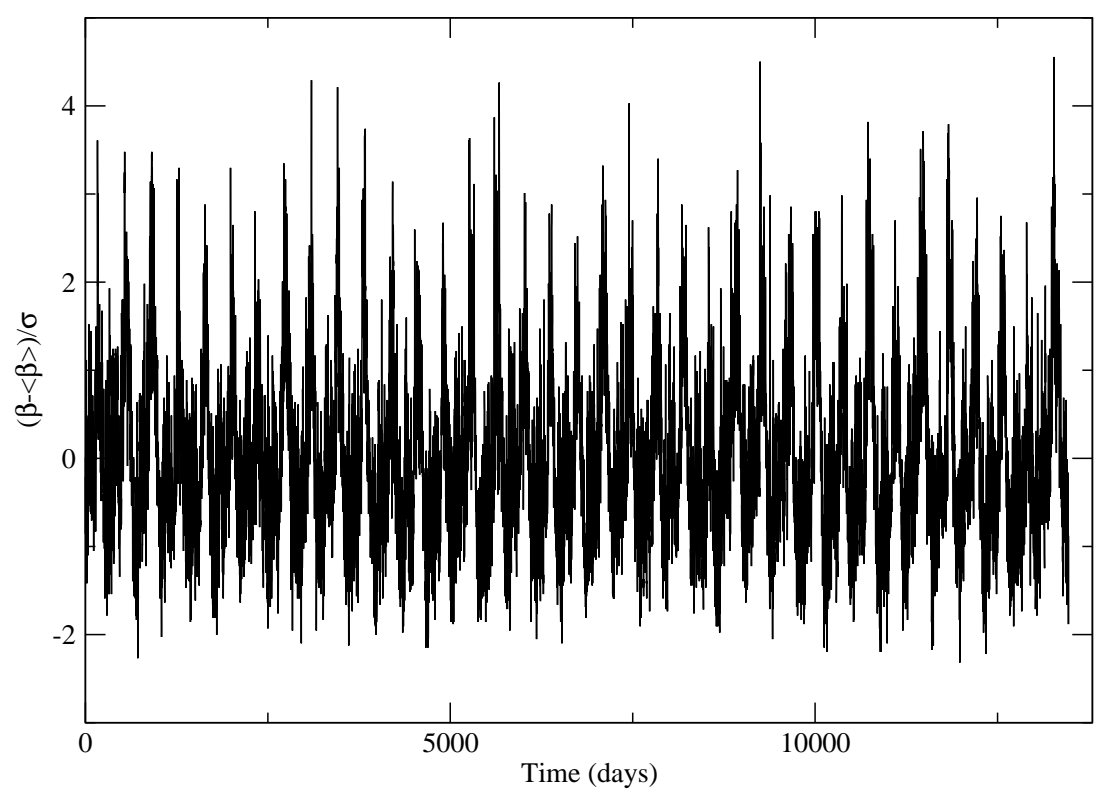

FIG. 13: Daily measured inverse mean temperature in Darwin 1975-2011 


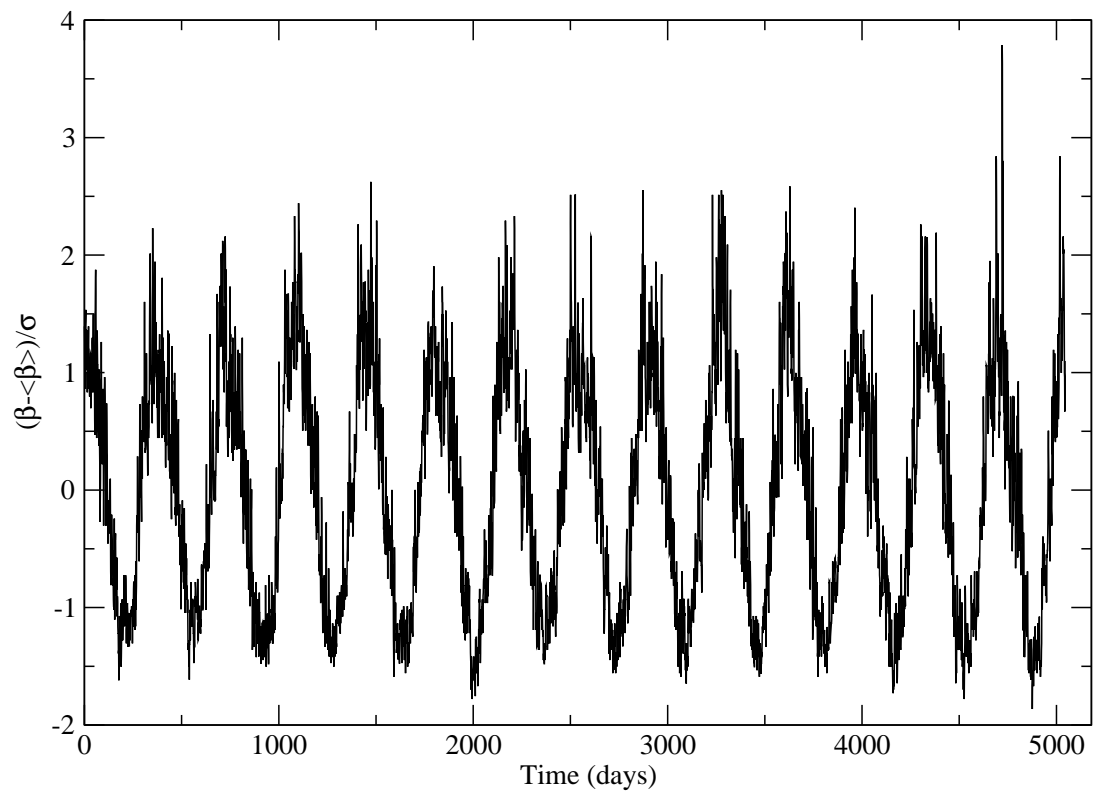

FIG. 14: Daily measured inverse mean temperature in Santa Fe 1998-2011

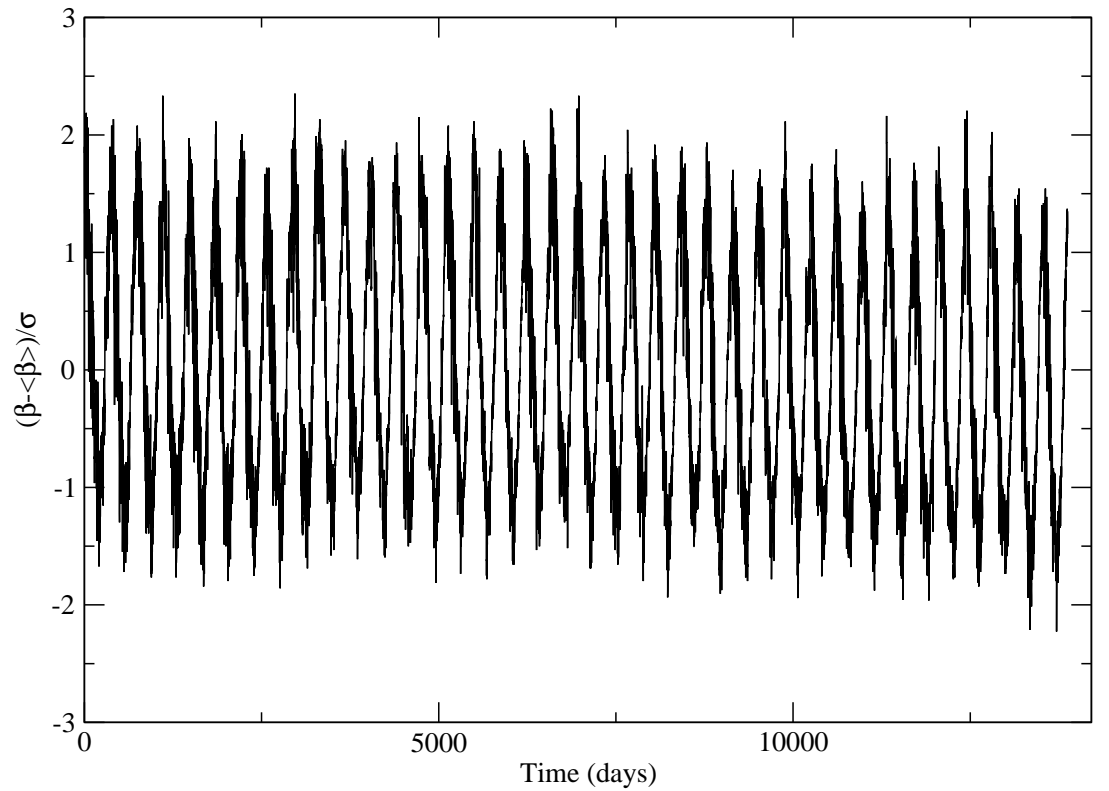

FIG. 15: Daily measured inverse mean temperature in Dubai 1974-2011 


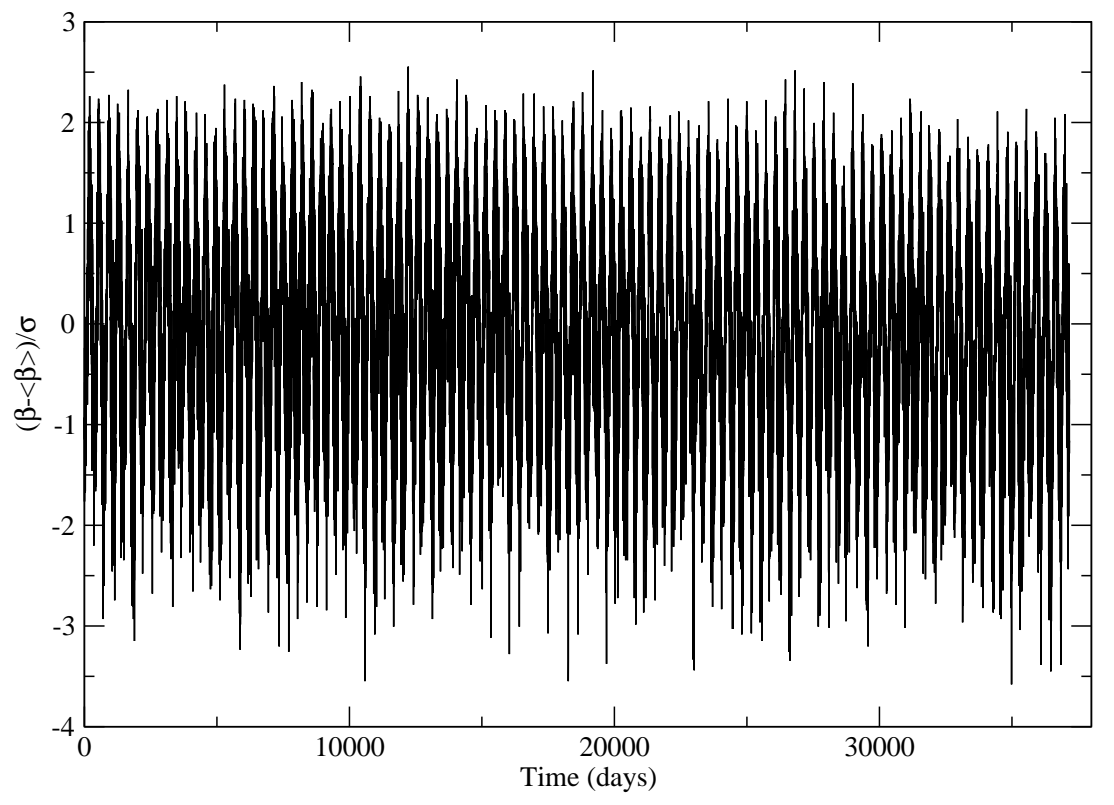

FIG. 16: Daily measured inverse mean temperature in Sydney 1910-2011

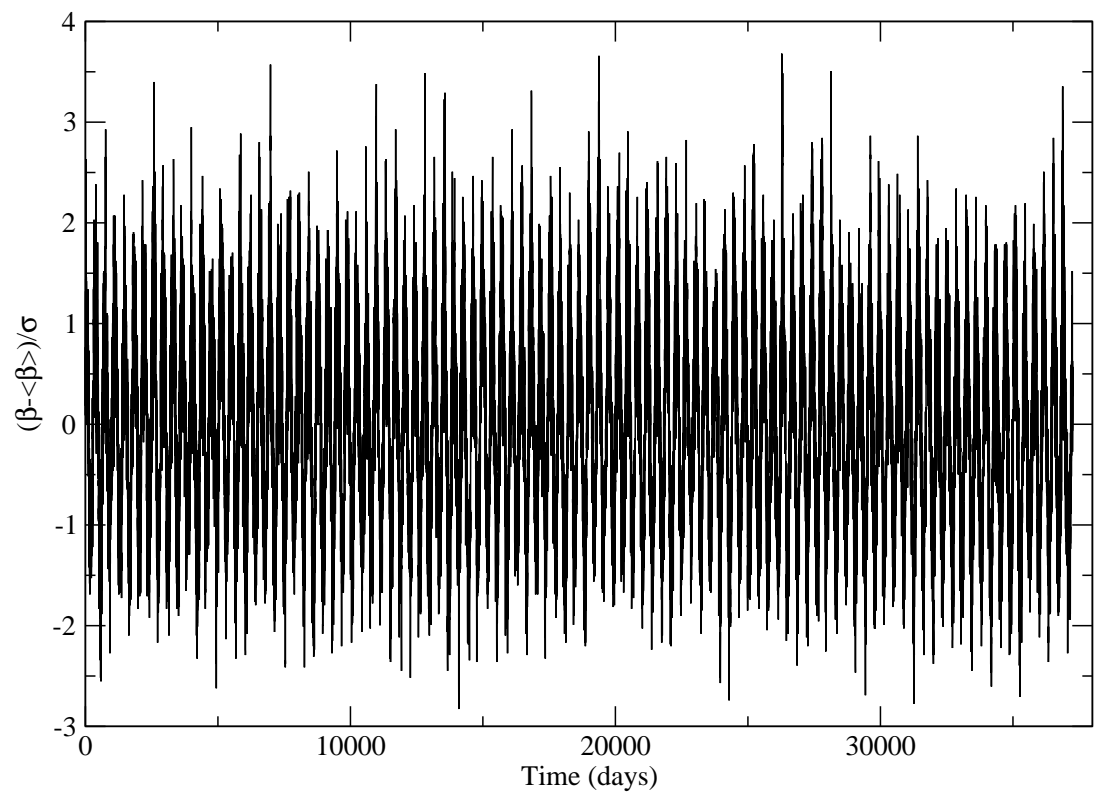

FIG. 17: Daily measured inverse mean temperature in Central England (Lancashire, London and Bristol) 1910-2011 


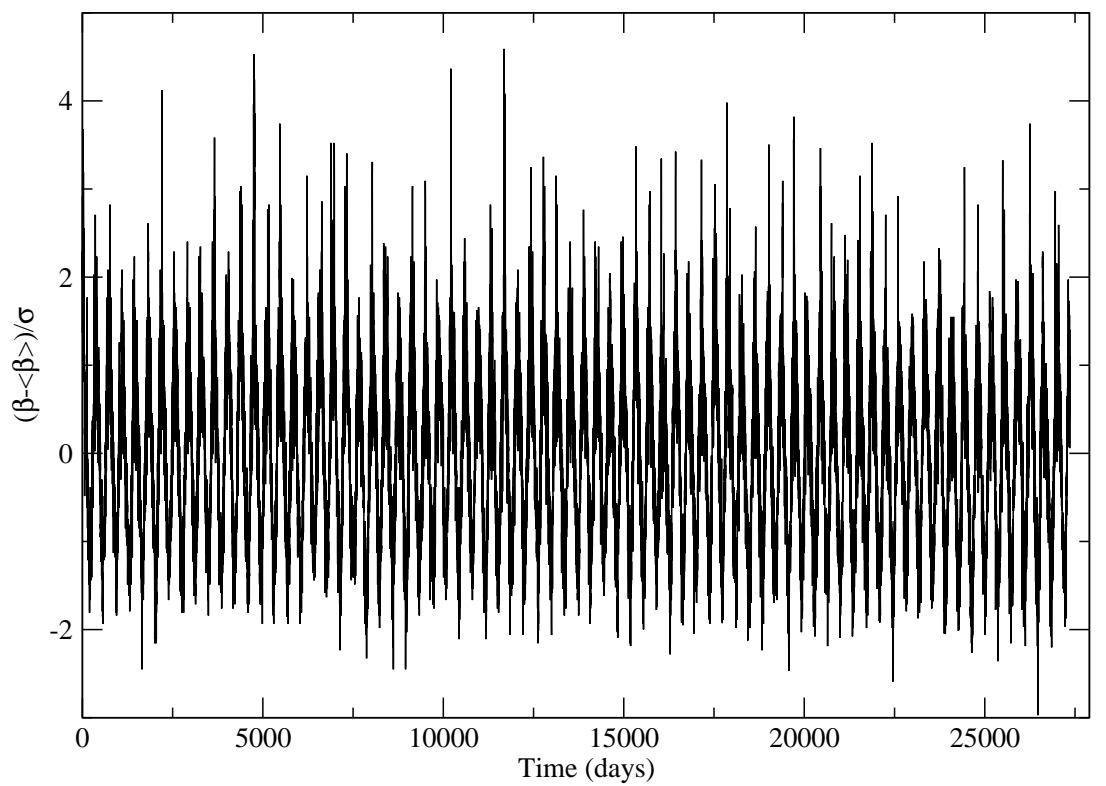

FIG. 18: Daily measured inverse mean temperature in Vancouver 1937-2011

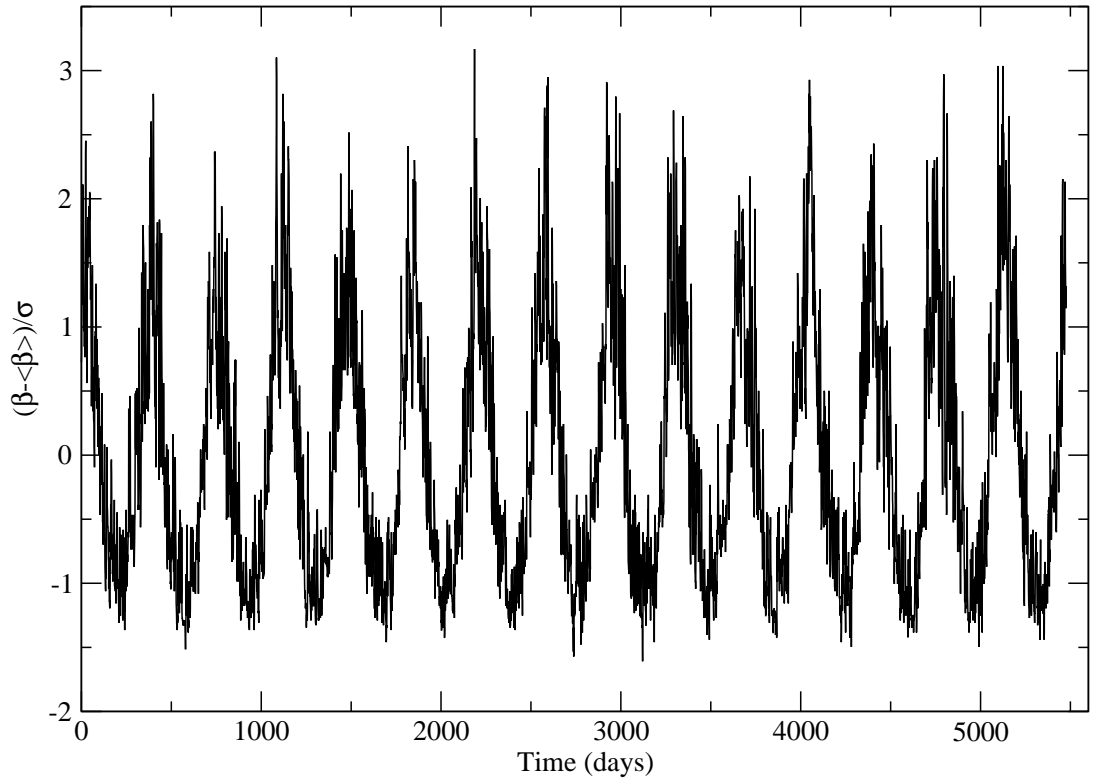

FIG. 19: Daily measured inverse mean temperature in Hong Kong 1997-2011 


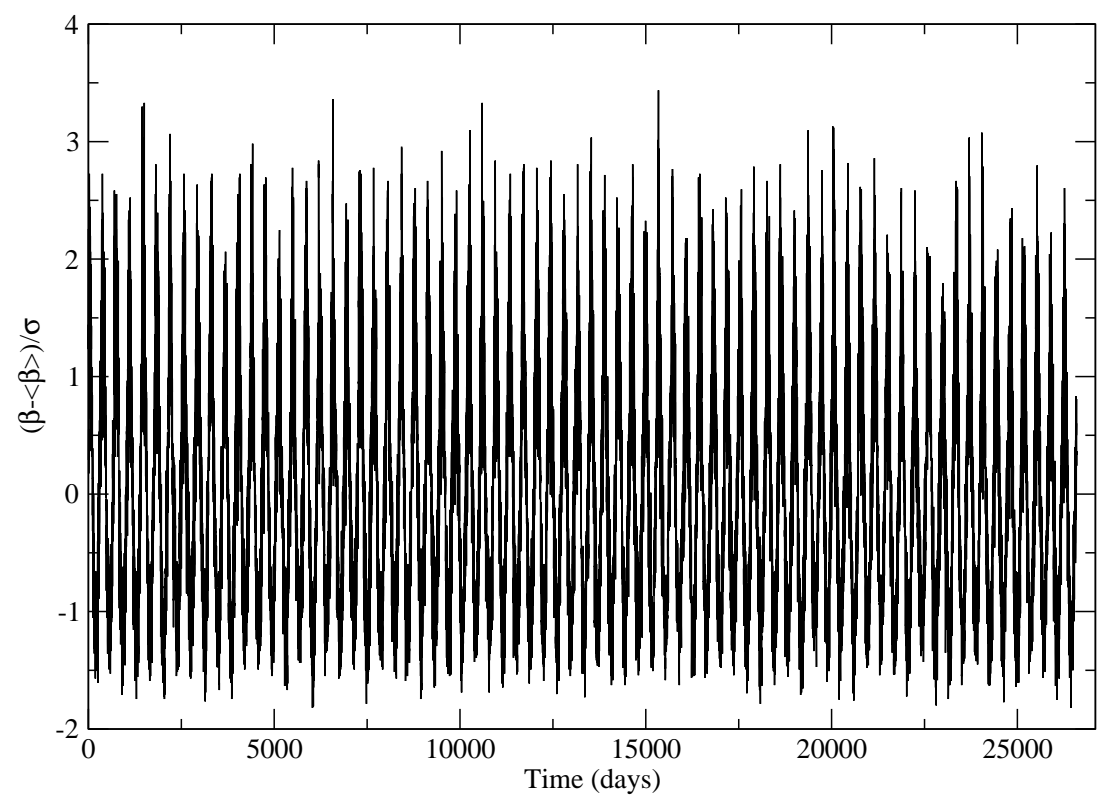

FIG. 20: Daily measured inverse mean temperature in Ottawa 1939-2011

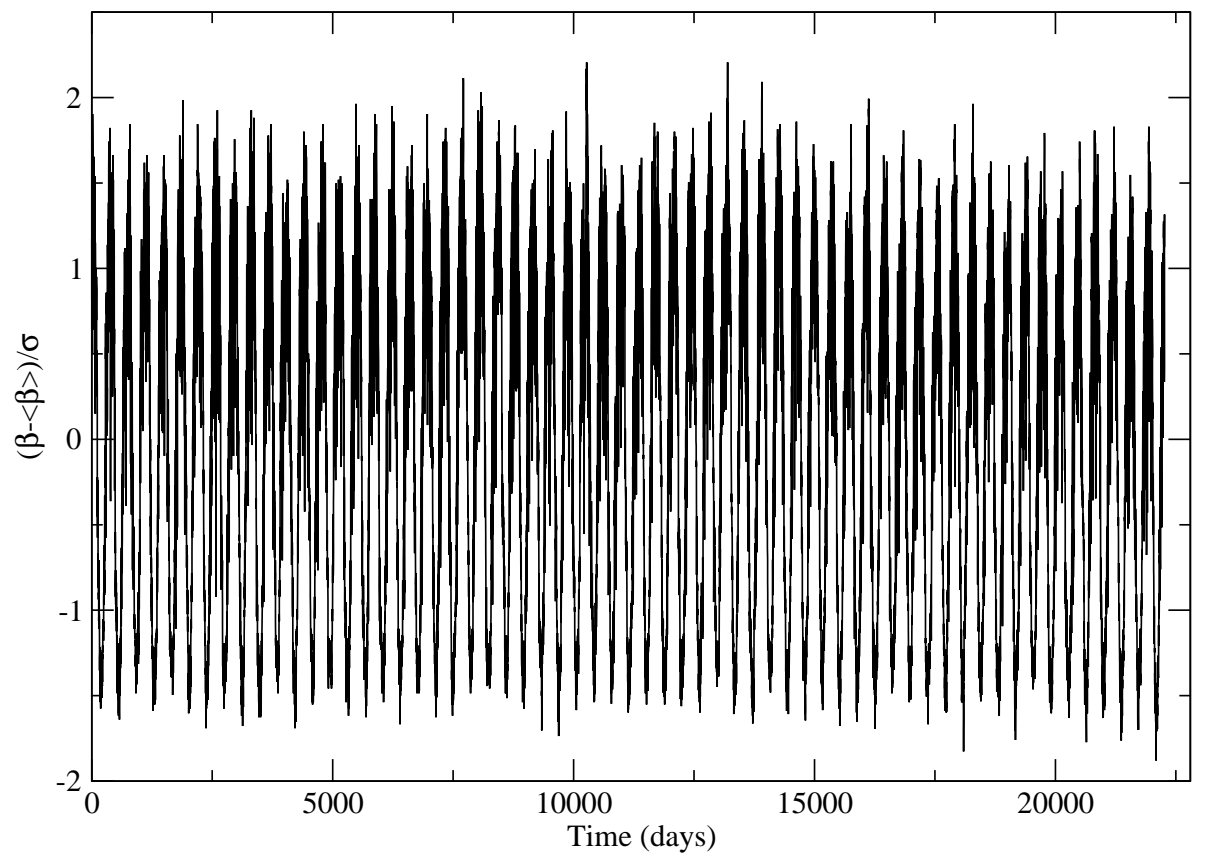

FIG. 21: Daily measured inverse mean temperature in Eureka 1951-2011

Figs. 22-30 show the histograms of inverse temperature time series for the various locations. These distributions are the ones relevant for the superstatistical description of thermodynamic devices that need to work in the open air and that are supposed to work properly for continuous operation over many years. Similarly, these are also the distributions seen by biological populations that don't have the luxury of artificial heating or air-conditioning. The details of the distribution of course heavily depend on the climate zone of the location. 


\begin{tabular}{|c|c|c|l|l|}
\hline Location and time period & Summer(Celcius) & Winter $($ Celcius $)$ & $\beta_{1}$ & $\beta_{2}$ \\
\hline Darwin (1975-2011) & 28.32 & - & 1.8 & - \\
\hline Santa Fe (1998-2011) & 22.06 & 3.31 & 18 & 4 \\
\hline Dubai (1974-2011) & 33.03 & 20.39 & 7 & 8 \\
\hline Sydney (1910-2011) & 20.22 & 13.78 & 2 & 3.5 \\
\hline Central England (1910-2011) & 14.26 & 7.12 & 3.6 & 1.4 \\
\hline Vancouver (1937-2011) & 15.80 & 7.05 & 5 & 2 \\
\hline Hong Kong (1997-2011) & 27.99 & 19.31 & 10 & 2.7 \\
\hline Ottawa (1939-2011) & 18.49 & -1.04 & 12 & 2.1 \\
\hline Eureka (1951-2011) & 4.21 & -36.3 & 43 & 5.5 \\
\hline
\end{tabular}

TABLE I: Maxima (in degree Celsius) and variance parameters $\beta_{1}, \beta_{2}$ of the two Gaussians $\sim e^{-\beta_{i}(\beta-\bar{\beta})^{2}}$ used in the fits.

A typical observation is now that the relevant distributions have a double-peak structure, at least for non-tropical locations. Broadly, the left peak (small $\beta$ ) corresponds to summer and the right peak (large $\beta$ ) to winter. The entire distribution can be very roughly regarded as a superposition of two Gaussians, with some intermediate behaviour between the peaks. This intermediate behaviour is more pronounced for geographical locations that have big differences between summer and winter temperatures.

Apparently, for climates with strong differences of winter and summer temperatures the two peaks of our Gaussian fits (Fig. 22-30) are far apart. Table 1 lists the two temperatures where the two maxima in the histogram occur. They are consistent with an average temperature observed during a couple of months corresponding to summer and winter, respectively, at the different geographical locations. Some special locations, for example Darwin in Fig. 22, show again deviations from Gaussian behavior for $f(\beta)$, with exponential-like tails for large $\beta$, and sub-Gaussian behavior for small $\beta$ (the red line in that figure is a Gaussian).

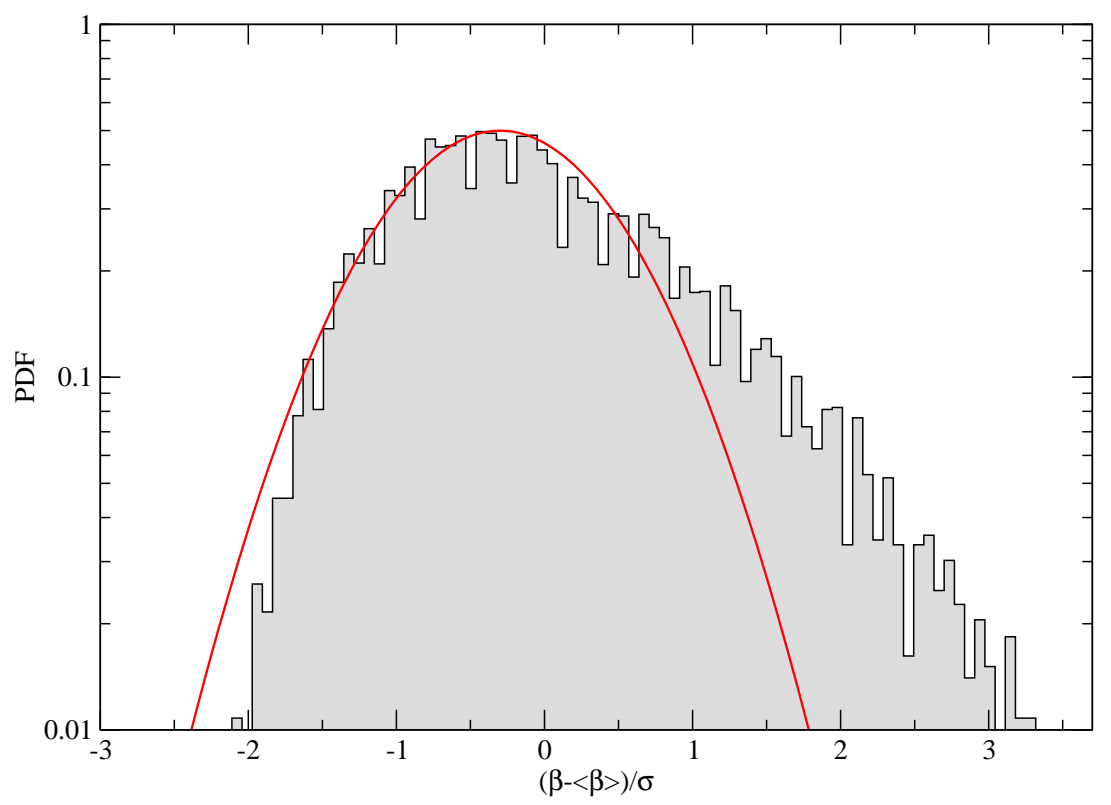

FIG. 22: Distribution of the daily measured inverse mean temperature in Darwin for 1975-2011 


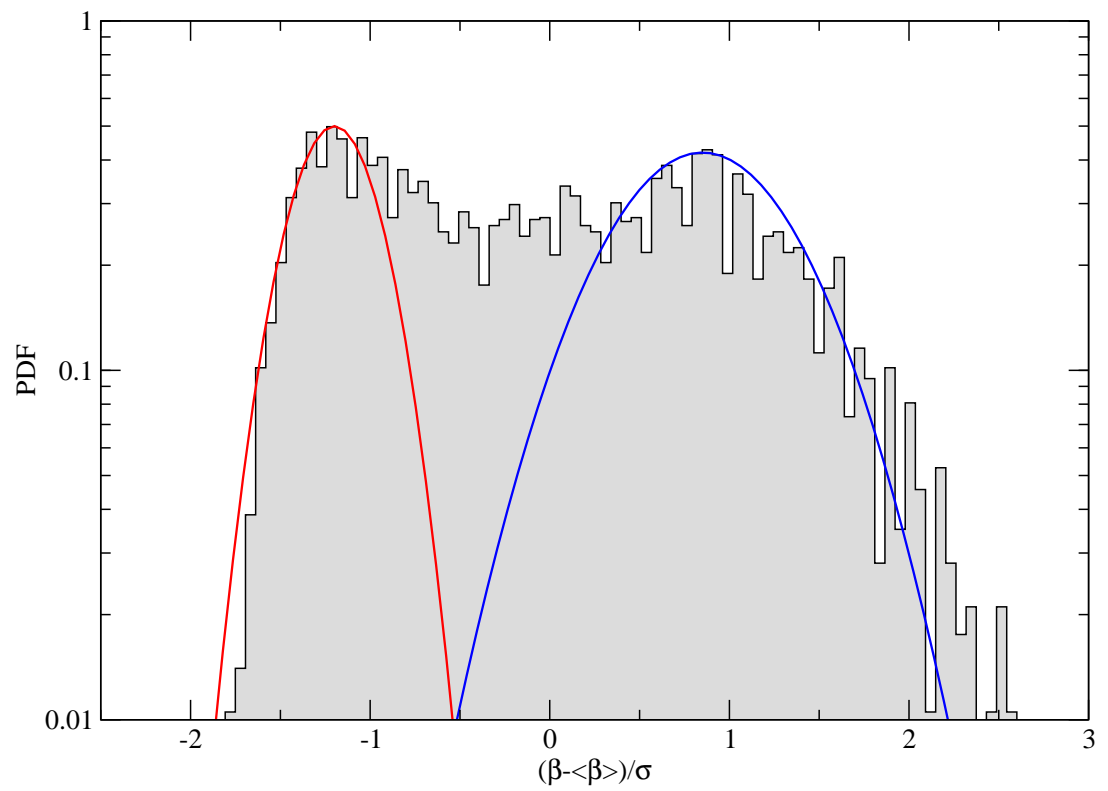

FIG. 23: Distribution of the daily measured inverse mean temperature in Santa Fe for 1998-2011

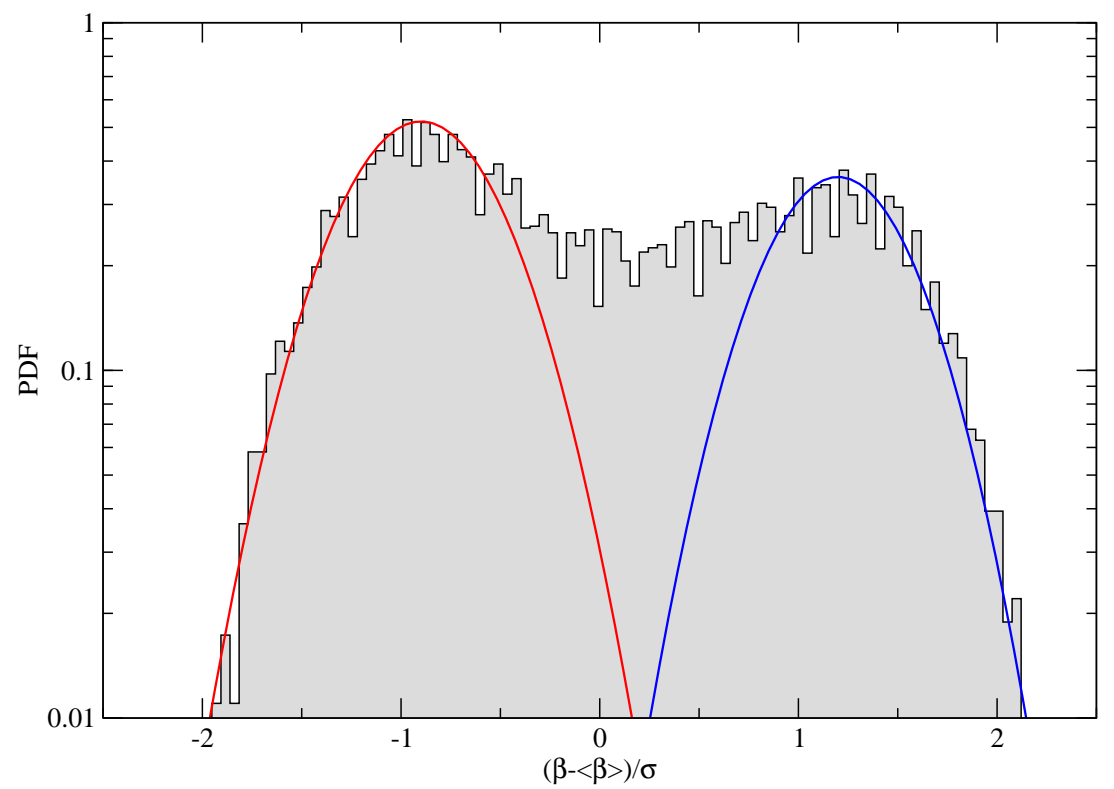

FIG. 24: Distribution of the daily measured inverse mean temperature in Dubai for 1974-2011 


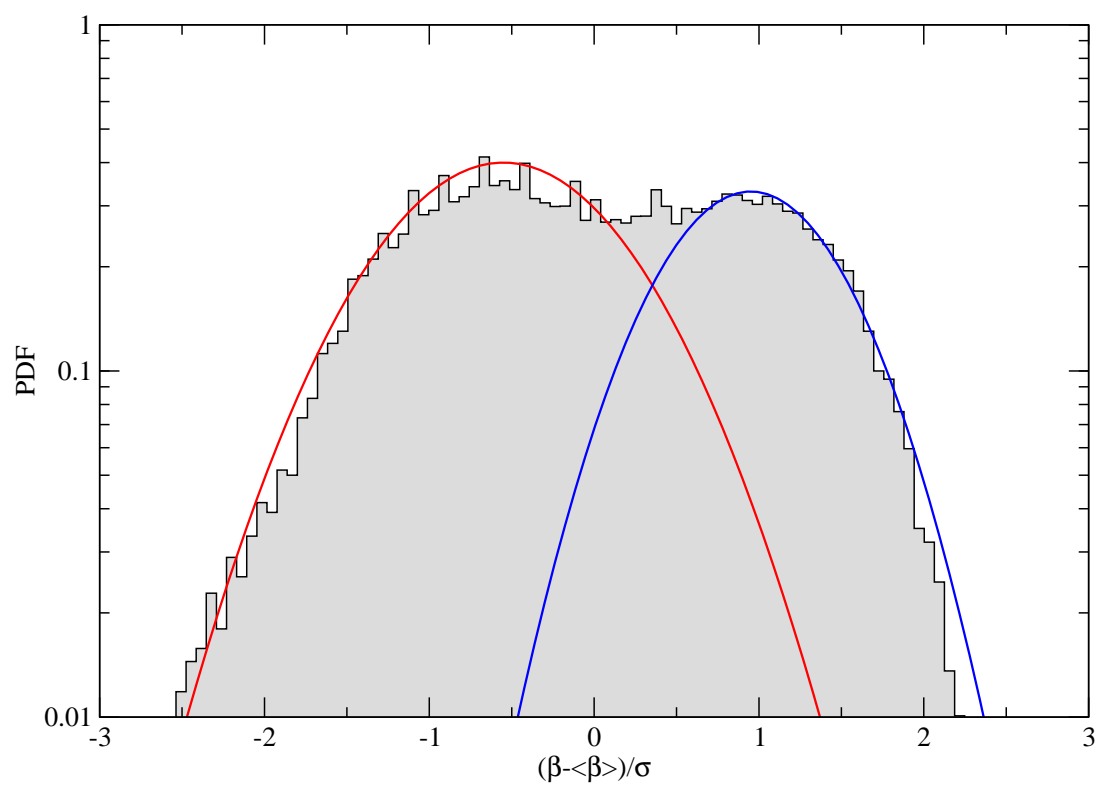

FIG. 25: Distribution of the daily measured inverse mean temperature in Sydney for 1910-2011

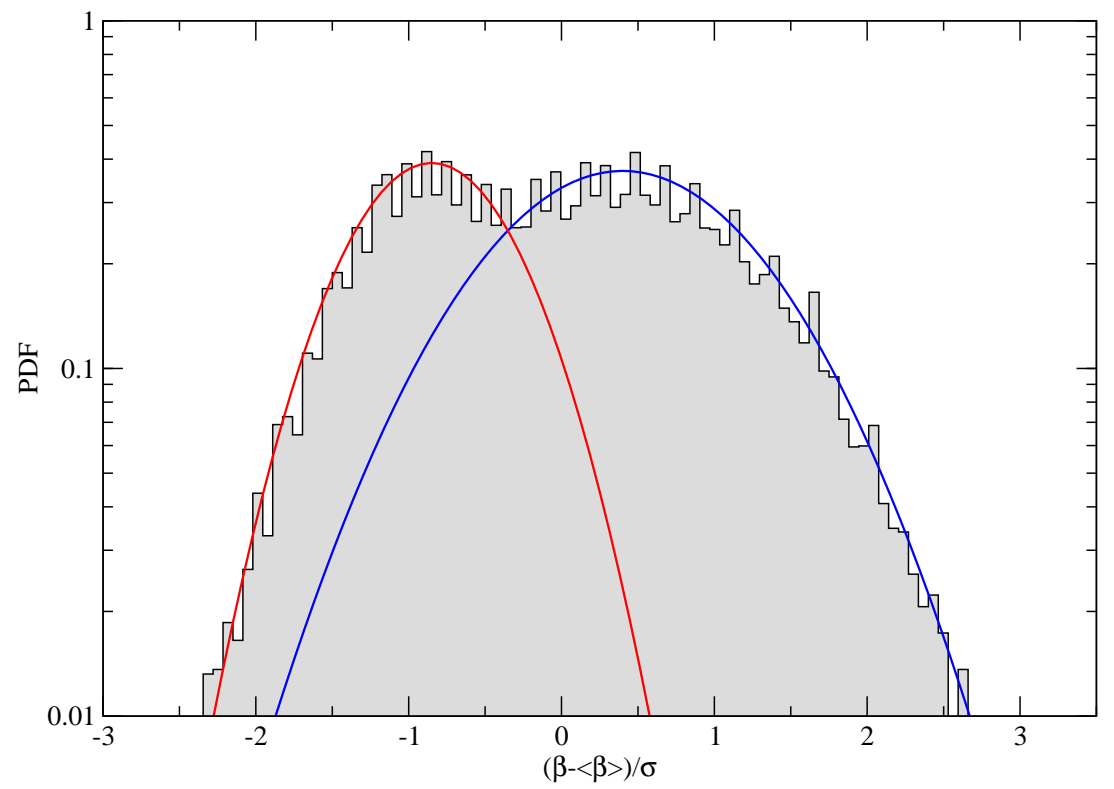

FIG. 26: Distribution of the daily measured inverse mean temperature in Central England (Lancashire, London and Bristol) for $1910-2011$ 


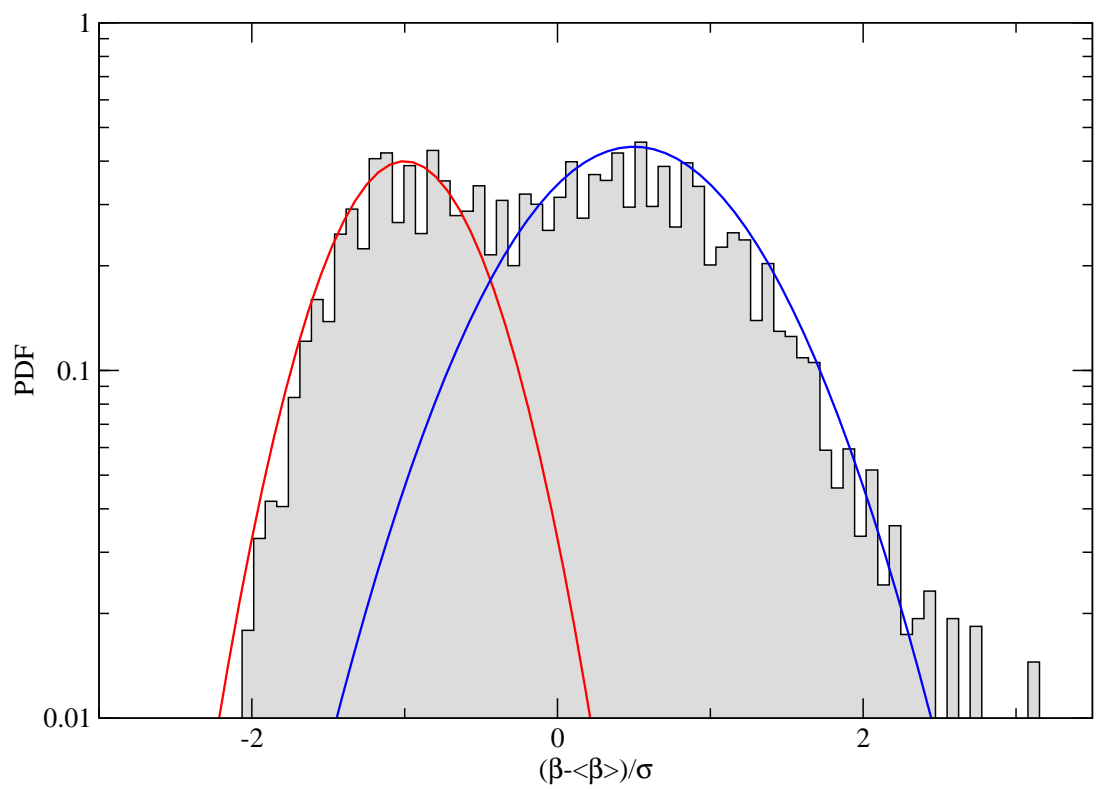

FIG. 27: Distribution of the daily measured inverse mean temperature in Vancouver for 1937-2011

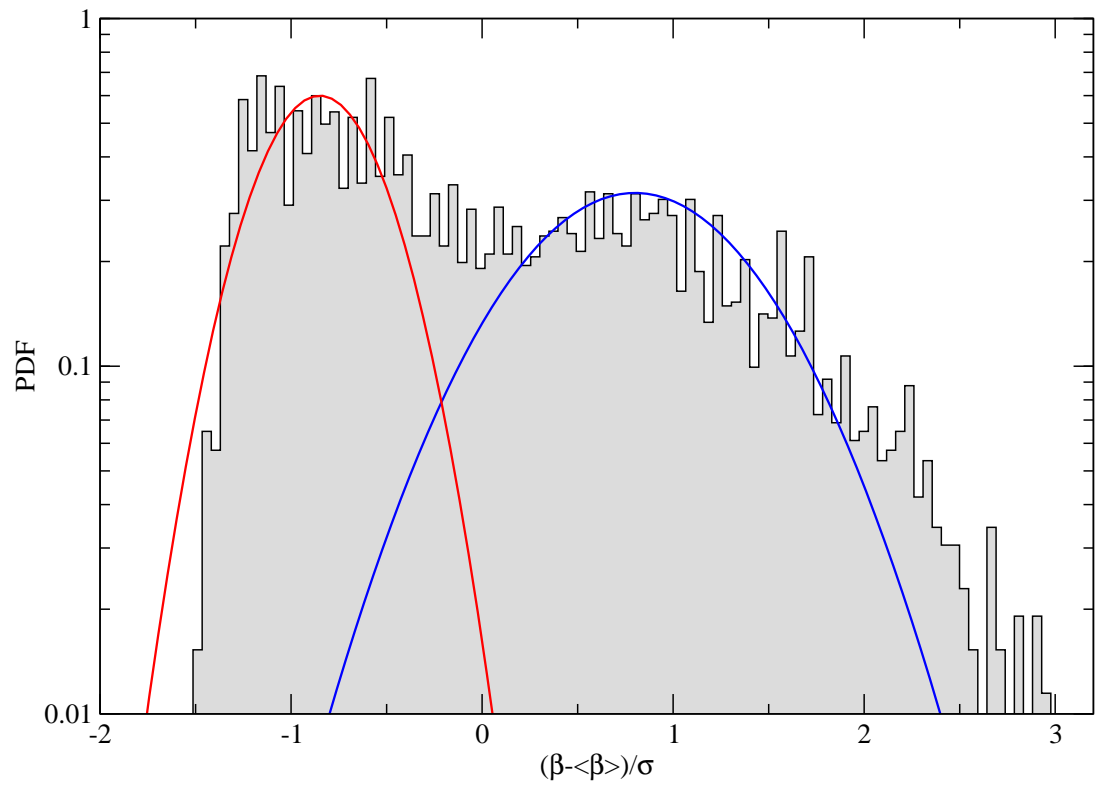

FIG. 28: Distribution of the daily measured inverse mean temperature in Hong Kong for 1997-2011 


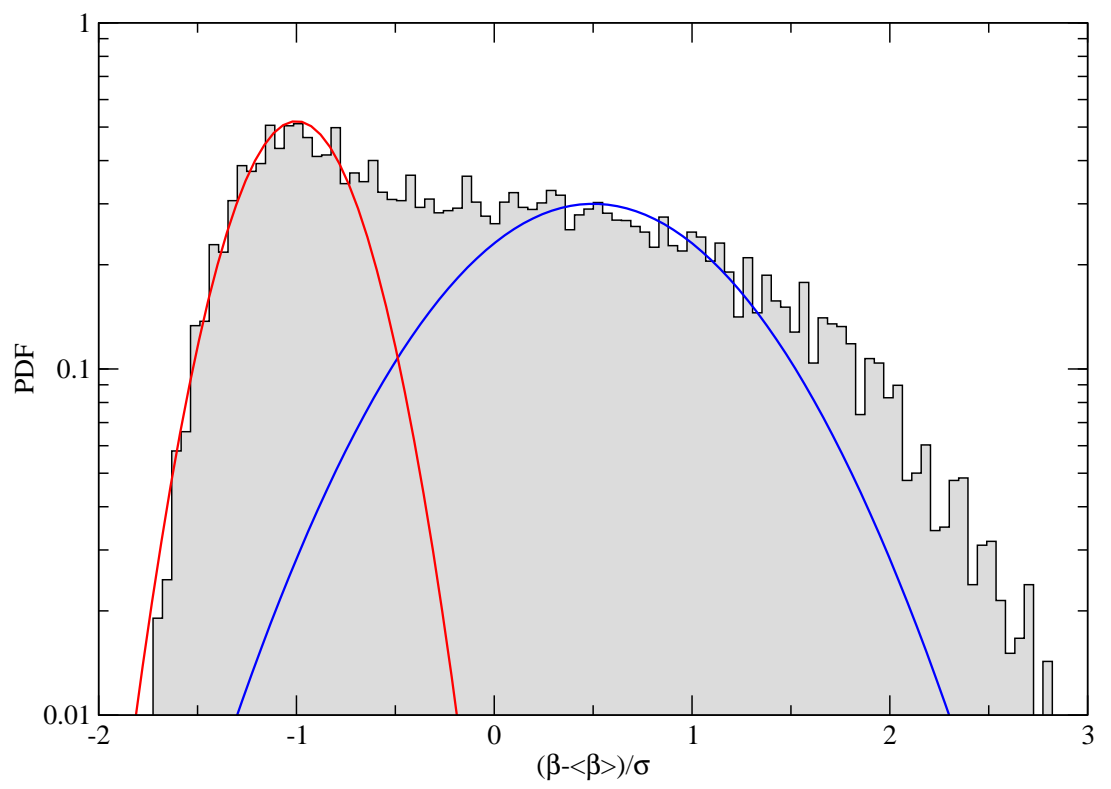

FIG. 29: Distribution of the daily measured inverse mean temperature in Ottawa for 1939-2011

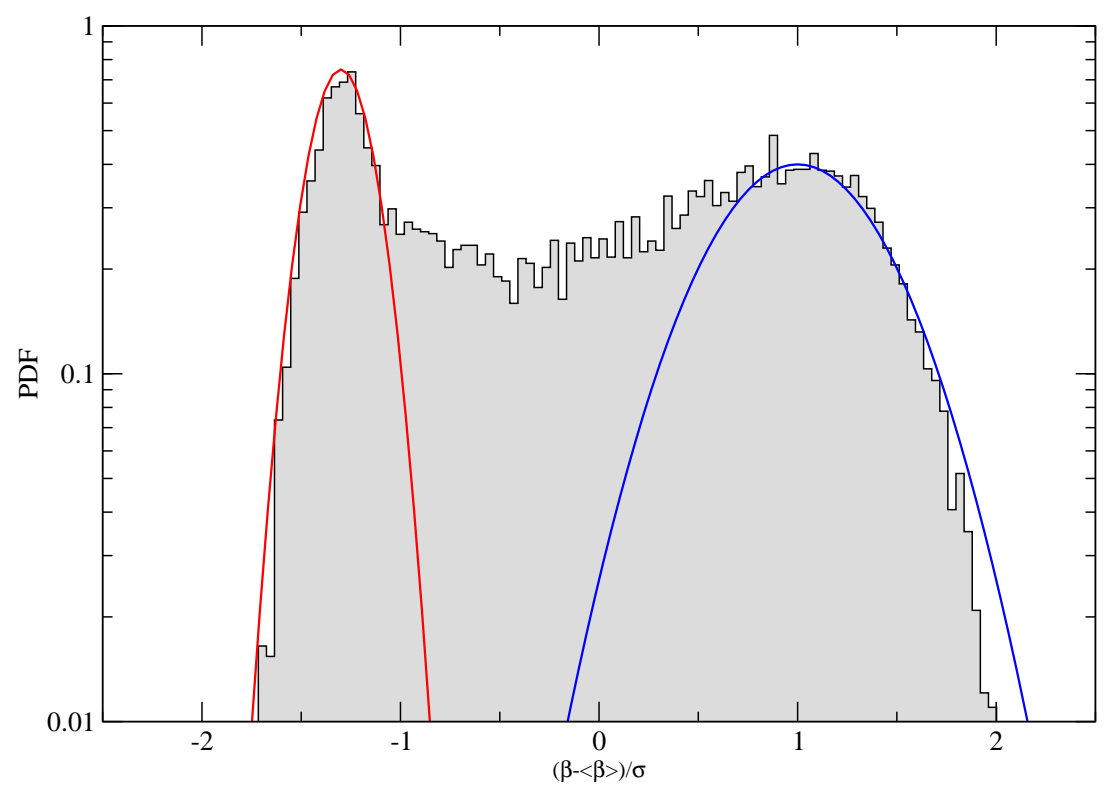

FIG. 30: Distribution of the daily measured inverse mean temperature in Eureka for 1951-2011

One may try to fit the data by other functional forms than a superposition of two Gaussians. For example, the histogram of daily measured inverse mean temperature in Dubai 1974-2011 (Fig. 31) is well fitted by an exponential $e^{-V(\beta)}$ of a double-well potential $V(x) \sim\left(C_{2} x^{2}+C_{3} x^{3}+C_{4} x^{4}\right)$. Generally, however, there is no simple analytic expression for the environmentally relevant inverse temperature distributions. 


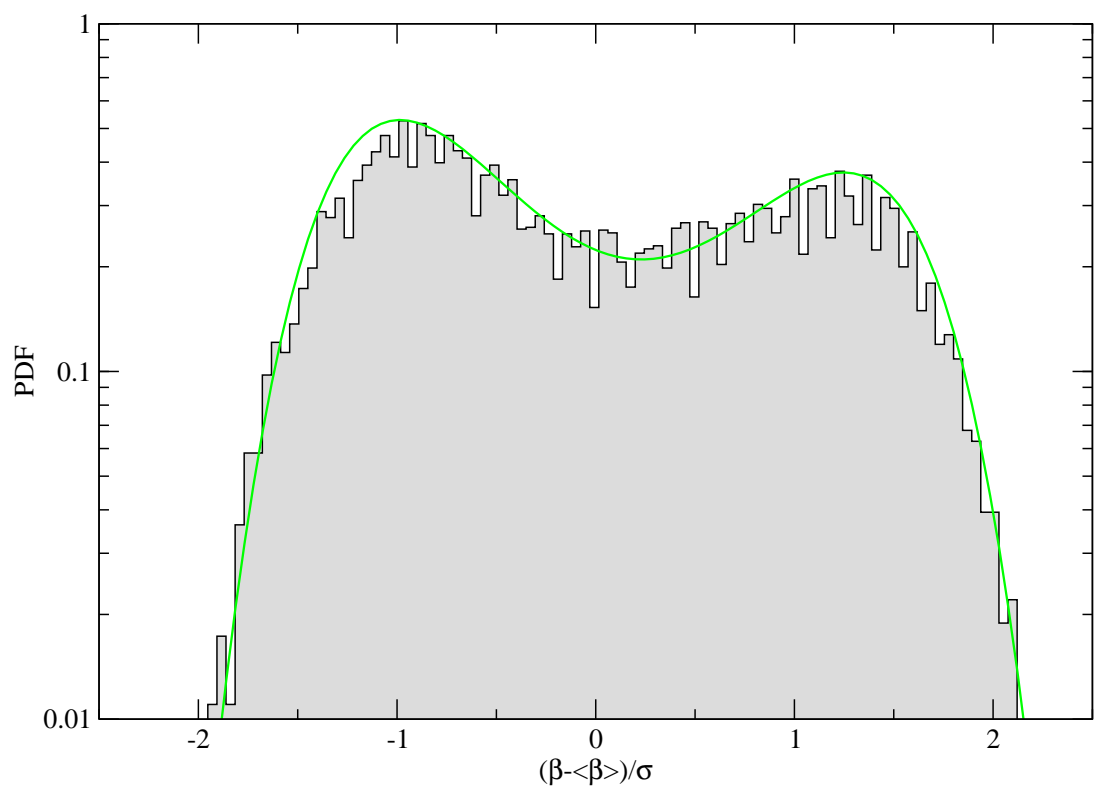

FIG. 31: Fit with the exponential of a double-well potential to the histogram of the daily measured inverse mean temperature in Dubai 1974-2011

\section{INTERPRETATION OF RESULTS IN TERMS OF KÖPPEN-GEIGER CLIMATE CLASSIFICATION SYSTEM}

Our superstatistical distributions are consistent with the Köppen-Geiger climate classification system [35 37] which is one of the most widely used climate classification systems. In the following we will briefly review this scheme and then interpret our long-term superstatistical distributions in the Köppen-Geiger context. Of course, so far our investigation only involves temperature distributions. In future work, we intend to take into account rainfall data as well [26, 27], which can also be modeled using superstatistical techniques.

There are basically 5 different climatic groups in the Köppen-Geiger scheme: Group A Tropical climates, group B Arid climates, group C Temperate climates, group D Cold climates and group E Polar climates. We have chosen at least one example in each of these groups: A: Darwin, B: Santa Fe, Dubai C: Sydney, Central England, Hongkong D: Ottawa, E: Eureka.

A second and third letter give more specific information within each of the groups. In our examples, we have collected data for the following climate types:

Aw (Tropical-Savannah): Darwin,

BSk (Arid-Steppe-Cold): Santa Fe,

BWh (Arid-Desert-Hot): Dubai,

Cfa (Temperate-Without dry season-Hot summer): Sydney,

$\mathrm{Cfb}$ (Temperate-Without dry season-Warm summer): Central England and Vancouver,

Cwa (Temperate-Dry winter-Hot summer): Hong Kong,

Dfb (Cold-Without dry season-Warm summer): Ottawa,

ET(Polar-Tundra): Eureka.

We summarize below short characterizations of these subtypes:

Aw (Tropical-Savannah) subtype is characterized by the coldest month which has a temperature of $18^{\circ} \mathrm{C}$ or more. There is no rainforest and the precipitation of the driest month is less than 100 - mean annual precipitation $/ 25$.

BSk (Arid-Steppe-Cold) subtype is characterized by mean annual precipitation equal or bigger than five times and less than ten times of the precipitation threshold. The mean annual temperature is less than $18^{\circ} \mathrm{C}$.

BWh (Arid-Desert-Hot) subtype is characterized by mean annual precipitation equal or less than five times of precipitation threshold. The mean annual temperature is equal or bigger than $18^{\circ} \mathrm{C}$.

Cfa (Temperate-Without dry season-Hot summer) subtype is characterized by the temperature of the hottest month being equal and bigger than $22^{\circ} \mathrm{C}$ and the temperature of the coldest month is between $0^{\circ} \mathrm{C}$ and $18^{\circ} \mathrm{C}$. There is no dry season for summer and winter.

Cfb (Temperate-Without dry season-Warm summer) subtype is characterized as follows: The temperature of the hottest month is bigger than $10^{\circ} \mathrm{C}$ and the temperature of the coldest month is between $0^{\circ} \mathrm{C}$ and $18^{\circ} \mathrm{C}$. There is no 
dry season for summer and winter.There is no hot summer. The number of months where the temperature is above $10^{\circ} \mathrm{C}$ is equal or bigger than 4 .

Cwa(Temperate - Dry winter - Hot summer): The temperature of the hottest month is equal or bigger than $22^{\circ} C$. The temperature of the coldest month is between $0^{\circ} \mathrm{C}$ and $18^{\circ} \mathrm{C}$. Precipitation of the driest month in winter is less than one-tenth of precipitation of the wettest month in summer.

$\mathrm{Dfb}$ (Cold-Without dry season-Warm summer): The temperature of the hottest month is larger than $10^{\circ} \mathrm{C}$. The temperature of the coldest month is equal or less than $0^{\circ} \mathrm{C}$. There is no dry season for summer and winter and there is no hot summer. The number of months where the temperature is above $10^{\circ} \mathrm{C}$ is equal or bigger than 4 .

ET (Polar-Tundra) subtype is characterized by the temperature of the hottest month being between $0^{\circ} \mathrm{C}$ and $10^{\circ} \mathrm{C}$.

When looking at Fig. 22-30 and Table 1, the observed inverse temperature distributions are clearly consistent with the climate classifications of the various locations. The plot for Darwin (Fig. 22) is the only one which has only a single maximum, occuring at the very high temperature $28^{\circ} \mathrm{C}$, consistent with the tropical location and the non-existence of seasonal variations. Still it is interesting to see that the distribution strongly deviates from a Gaussian, there is a pronounced tail corresponding to large $\beta$.

All other plots exhibit a double-peak structure, corresponding to summer and winter. However, it is interesting that the variance of the Gaussians that we use for the fits can be very different. For example, for Santa Fe (Fig. 23) the summer-Gaussian (on the left) has a smaller variance than the winter-Gaussian (on the right), whereas for Dubai (Fig. 25) both Gaussians have about the same variance, and finally for Sidney (Fig. 26) the summer-Gaussian (on the left) has bigger variance than the winter-Gaussian (on the right). In this way one may use our plots to provide a quantitative characterization of Köppen-Geiger subtypes, or even a new classification.

The plots of Fig. 26 (Central England) and Fig. 27 (Vancouver) are very similar. This is to be expected, since both locations fall into the same climate type $\mathrm{Cfb}$.

The locations of the most likely temperatures (i.e. the maxima) observed in Fig. 28-30 are consistent with typical summer and winter temperatures in Hong Kong, Ottawa and Eureka. Note that these temperatures are averaged over day and night.

As already mentioned, for tropical locations, such as Darwin (Fig. 22), the two peaks merge in a single peak, as expected for regions where there is hardly any difference between summer and winter temperatures. On the other hand, for polar locations, the two peaks are strongly separated, as shown in Fig. 30. for the example of Eureka. Here the two Gaussians are very far apart from each other. We suggest to use the variance of the winter- and summer-Gaussians, as well as their distance, as useful quantitative measures to characterize the temperature profile of various climate types. For example, Sydney seems special since it is the only example where the summer-Gaussian has significantly higher variance than the winter-Gaussian. Dubai seems special as well, since both winter and summer-Gaussian seem to have roughly the same variance. This allows for an alternative fit with the exponential of a double-well potential (Fig. 31).

\section{SUPERSTATISTICS WITH DOUBLE-PEAKED DISTRIBUTIONS}

The original concept of superstatistics provided an approximation method for sharply peaked temperature distributions, which resulted in a perturbative expansion around the Boltzmann-Gibbs limiting case [1]. Apparently, environmental superstatistics does not have sharply peaked distributions $f(\beta)$, but as we saw in the previous section there are broad distributions that often have two maxima. Hence the effective Boltzmann factors $B(E)=\int f(\beta) e^{-\beta E} d \beta$ can only be evaluated numerically. One idea would be to effectively separate the two maxima and to do a superposition of two single-peaked superstatistics, one for the summer and one for the winter. This is particularly useful if the thermodynamic device is supposed to work in one season with a particular performance, and in the other season with another one. This concept basically boils down to consider conditional temperature distributions, conditioned on the season of the year.

Another interesting question one can ask for environmental superstatistics is the large-energy asymptotics of the generalized Boltzmann factors $B(E)$. One can apply the techniques of [3] to determine, at least in principle, the decay of $B(E)$ with $E$ for large $E$. For this the small- $\beta$ behavior of $f(\beta)$ is relevant. For example, if $f(\beta) \sim \beta \gamma$ for small $\beta$, then the generalized Boltzmann factors decay with a power law for large $E$, the power law exponent being related to $\gamma[3]$ :

$$
B(E) \sim E^{-\gamma-1}
$$

Tsallis $q$-statistics [20, 21] is a particular example, with $q=\left\langle\beta^{2}\right\rangle /\langle\beta\rangle^{2}[1]$. In practice, for this one would need very precise data of $f(\beta)$ for small $\beta$, which in our environmental interpretation corresponds to the statistics of hot summers. The extreme-event statistics of very hot summers determines the asymptotic large- $E$ behaviour of the effective thermostatistics of the statistical mechanics device under consideration. Thus, to decide on the asymptotic 
behaviour of environmentally relevant $B(E)$ a systematic investigation of extreme events is needed, namely that of very hot summers.

\section{GLOBAL WARMING}

As we have already mentioned before, in some of our data one sees a systematic trend which can be associated with global warming. As it is well-known, global warming is the rise in the average temperature of the Earth's atmosphere and of the oceans since the late 19th century and its projected continuation. It is primarily caused by increasing concentrations of greenhouse gases produced by human activities such as the burning of fossil fuels and deforestation. It comes with sea level rises and widespread decreases in snow and ice extent.

Quantitatively, the Earth's average surface temperature rose by $0.74 \pm 0.18{ }^{\circ} \mathrm{C}$ over the period 1906-2005 [38 40]. The rate of warming over the last half of that period was almost double that of the period as a whole $\left(0.13 \pm 0.03^{\circ} \mathrm{C}\right.$ per decade, versus $0.07 \pm 0.02{ }^{\circ} \mathrm{C}$ per decade). The urban heat island effect is very small and accounts for less than $0.002{ }^{\circ} \mathrm{C}$ of warming per decade since 1900 [38]. Temperatures in the lower troposphere have increased between 0.13 and $0.22{ }^{\circ} \mathrm{C}\left(0.22\right.$ and $\left.0.4^{\circ} \mathrm{F}\right)$ per decade since 1979 , according to satellite temperature measurements [39]. Arctic regions are especially vulnerable to the effects of global warming, as has become apparent in the melting sea ice in recent years. Climate models predict much greater warming in the Arctic than the global average [40].

If sampled over many decades, the precision of some of our data is good enough to reveal the effects of global warming. As an example we show in Fig. 32 the average of daily measured mean temperature of every single year in Eureka, Nunavut, Canada from 1951-2011. This is an arctic region and our plot shows that the average temperature grows by $0.92{ }^{\circ} \mathrm{C}$ per decade (Fig. 33). So in this polar region the warming occurs at a much higher rate than the global warming rate that is averaged over the entire earth.

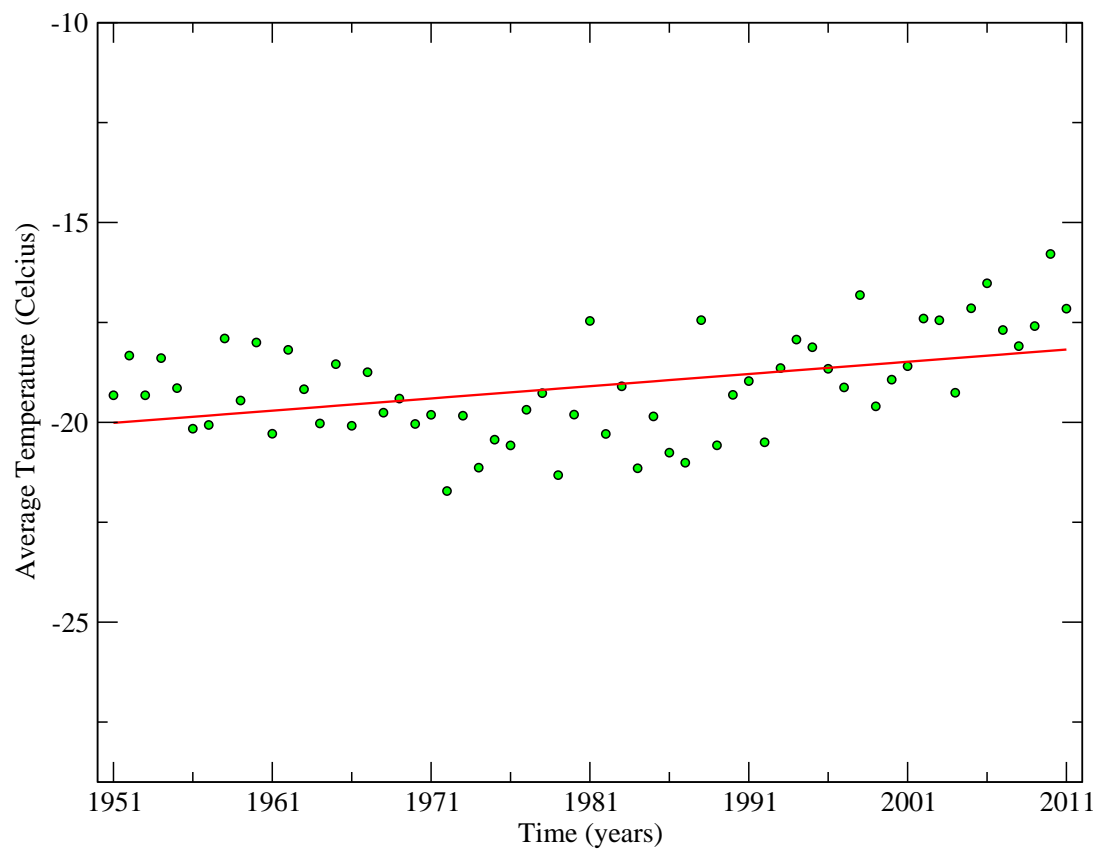

FIG. 32: Average of daily measured mean temperature of every single year in Eureka 1951-2011 


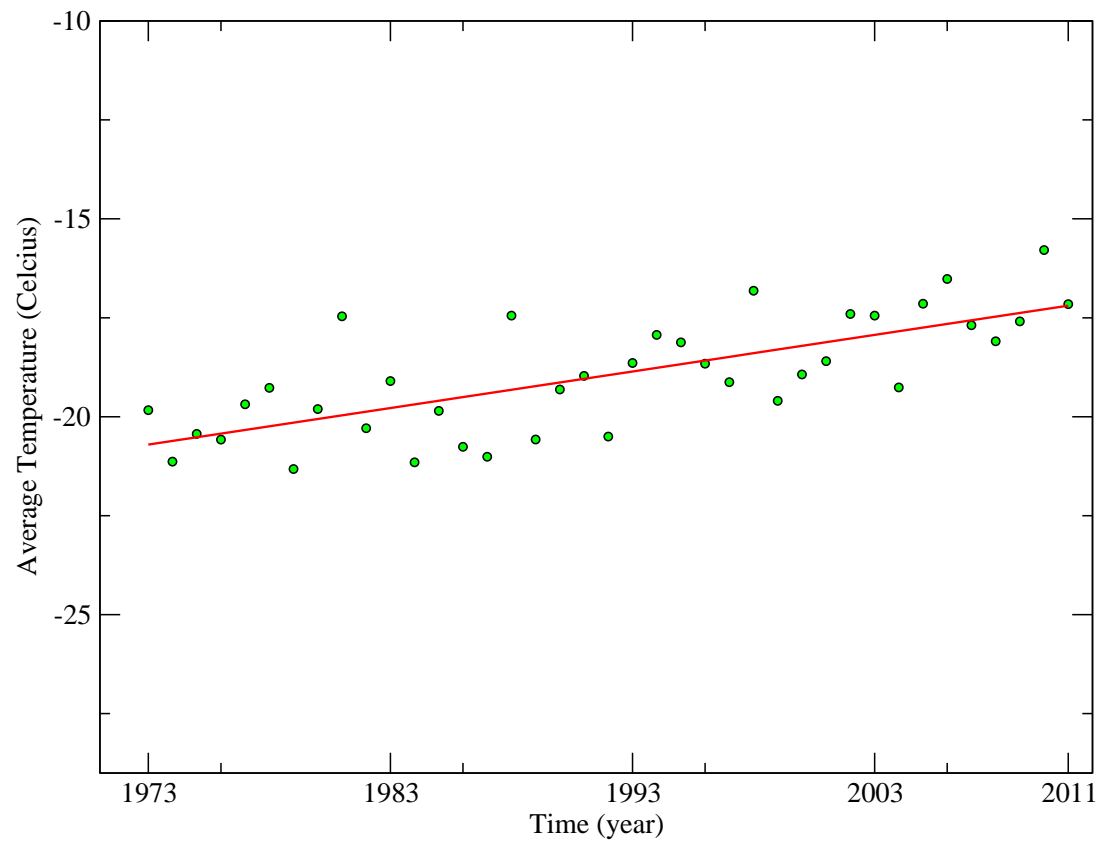

FIG. 33: Average of daily measured mean temperature of every single year in Eureka 1973-2011

For some of the other locations we also observe a systematic increase in average temperature (Figs. 34-37). For Dubai (Fig. 34) the rate is also rather high, $0.63{ }^{\circ} \mathrm{C}$ per decade. For Sydney (Fig. 35), Vancouver (Fig. 36), and Ottawa (Fig. 37) one has rates comparable to the average global warming rate, namely $0.13,0.11$ and $0.18{ }^{\circ} \mathrm{C}$ per decade, respectively. It is in the remit of the superstatistics approach that it can very well model both stochastic effects as well as systematic drifts, as long as there is a clear separation of time scales.

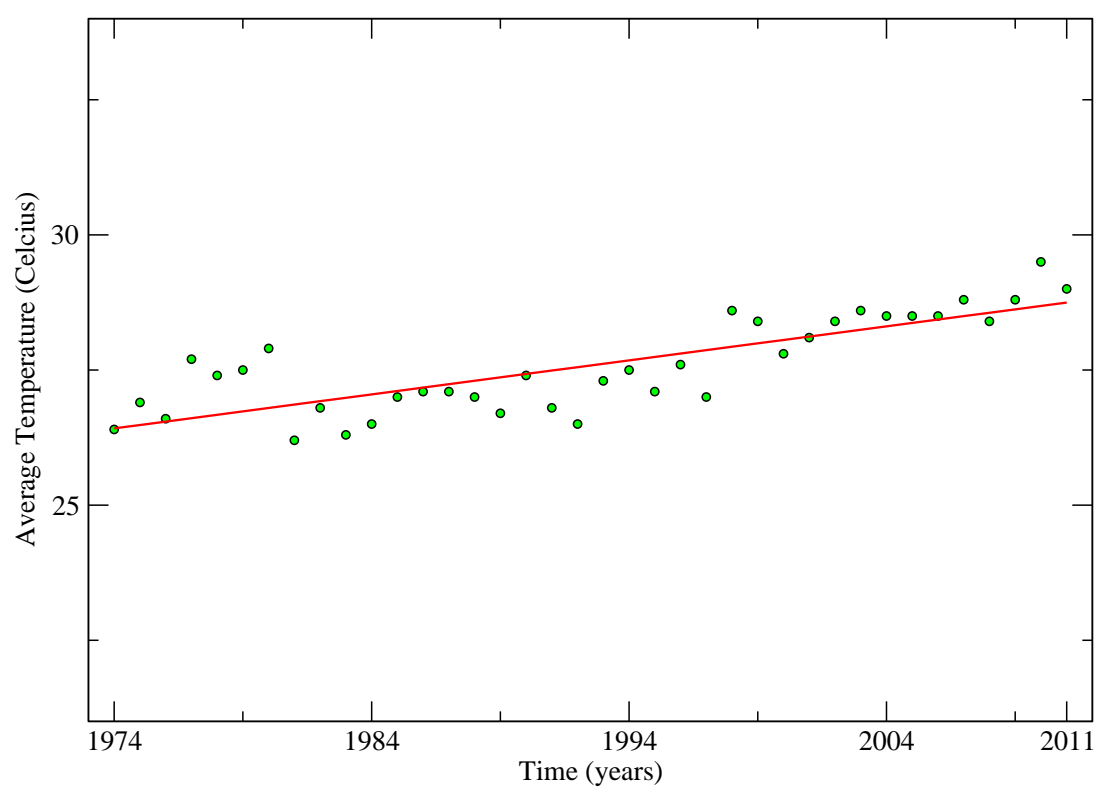

FIG. 34: Average of daily measured mean temperature of every single year in Dubai 1974-2011 


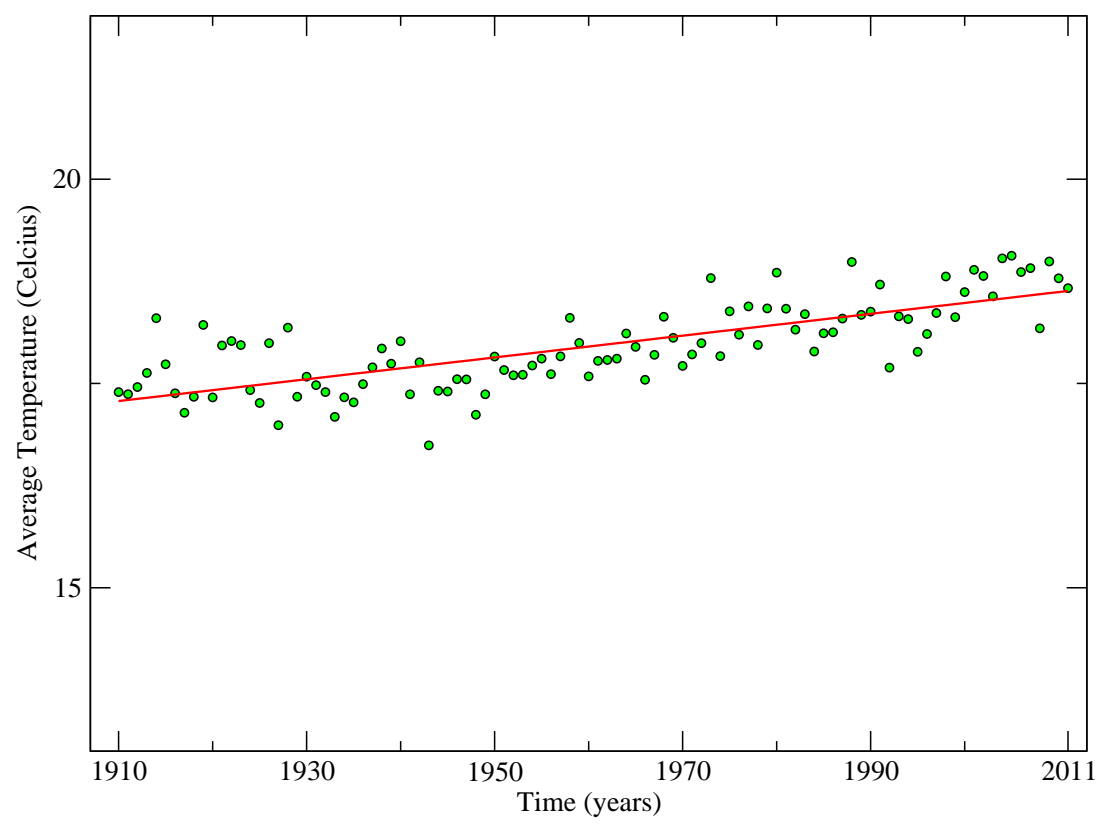

FIG. 35: Average of daily measured mean temperature of every single year in Sydney 1910-2011

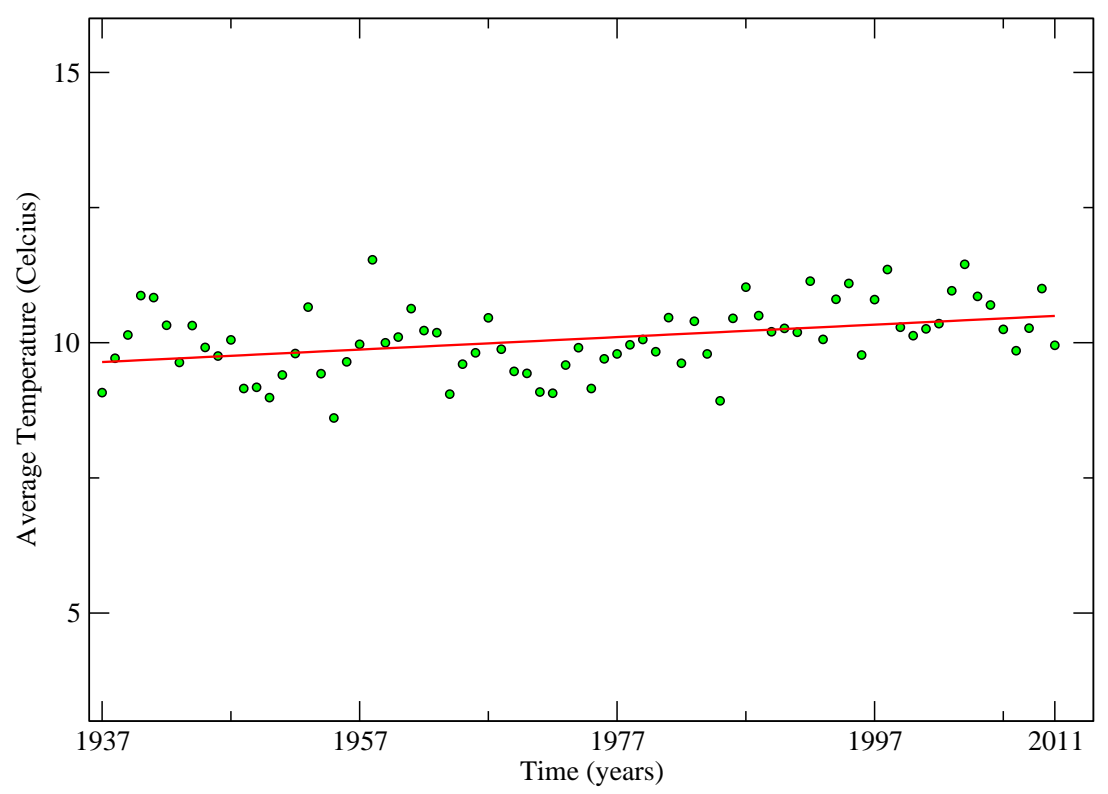

FIG. 36: Average of daily measured mean temperature of every single year in Vancouver 1937-2011 


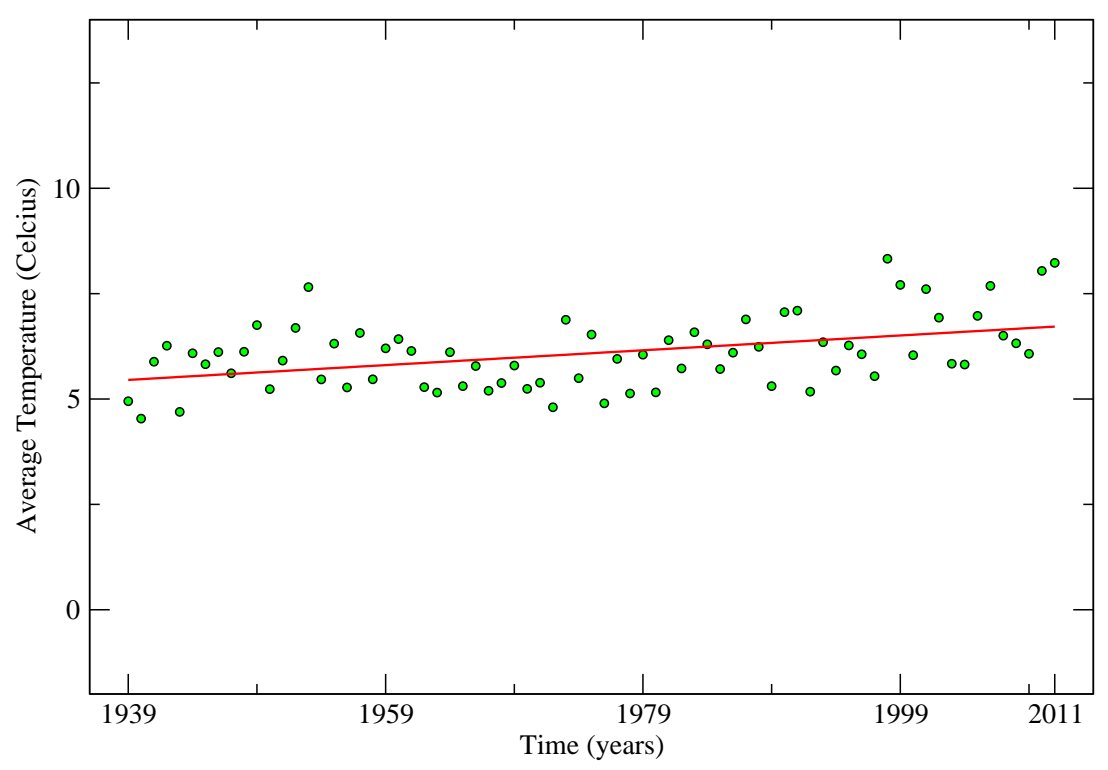

FIG. 37: Average of daily measured mean temperature of every single year in Ottawa 1939-2011

In the following we illustrate that global warming (i.e., a slight increase in average temperature) has an even more dramatic effect if the fluctuations of temperature at the various spatial locations are taken into account. To see this, let us consider a simple toy model, namely that of a $\chi$-square distribution of inverse temperature of degree $n$, as given by

$$
f(\beta)=\frac{1}{\Gamma\left(\frac{n}{2}\right)}\left(\frac{n}{2 \beta_{0}}\right)^{\frac{n}{2}} \beta^{\frac{n}{2}-1} e^{-\frac{n \beta}{2 \beta_{0}}} .
$$

Near the maximum, this distribution is close to a Gaussian distribution, for which we had evidence in section 2, provided we condition the observed temperatures onto a particular month of the year. For this toy model the average of the fluctuating $\beta$ is given by

$$
\langle\beta\rangle=\int_{0}^{\infty} \beta f(\beta)=\beta_{0}
$$

and the variance by

$$
\left\langle\beta^{2}\right\rangle-\beta_{0}^{2}=\frac{2}{n} \beta_{0}^{2}
$$

Let us now couple this environmental temperature bath to a local system, which for simplicity we simply take to be an ideal gas, although more generally we may think of more complicated ecosystems influenced by the temperature fluctuations of its environment. The kinetic energy of a test particle is given by $E=\frac{1}{2} v^{2}$, and for a given inverse temperature the velocity $v$ is distributed according to the Gaussian distribution

$$
p(v \mid \beta)=\sqrt{\frac{\beta}{2 \pi}} e^{-\frac{1}{2} \beta v^{2}} .
$$

Taking into account the environmental temperature fluctuations, the long-term probability density to observe the velocity $v$ of the test particle is given by the marginal probability $p(v)$ as

$$
p(v)=\int_{0}^{\infty} f(\beta) p(v \mid \beta) d \beta
$$

which in our toy example case can be evaluated as

$$
p(v)=\frac{\Gamma\left(\frac{n}{2}+\frac{1}{2}\right)}{\Gamma\left(\frac{n}{2}\right)}\left(\frac{\beta_{0}}{\pi n}\right)^{\frac{1}{2}} \frac{1}{\left(1+\frac{\beta_{0}}{n} v^{2}\right)^{\frac{n}{2}+\frac{1}{2}}} .
$$


Indeed we obtain $q$-statistics [20, 21] where the entropic parameter $q$ is related to the number of degrees of freedom influencing the $\beta$-variable by $q-1=\frac{2}{n+1}$ [22]. More generally, also for other distributions $f(\beta)$, one may define a parameter $q$ measuring the strength of environmental inverse temperature fluctuations by [1]

$$
q=\frac{\left\langle\beta^{2}\right\rangle}{\langle\beta\rangle^{2}} .
$$

If there are no temperature fluctuations, i.e. $\beta=\beta_{0}=$ const, then $q=1$, otherwise $q>1$.

We are now in a position to quantitatively calculate the response to global warming, i.e. a slight decrease in the average inverse temperature $\beta_{0}$ for this superstatistical model system. Consider the mean kinetic energy associated with velocity $v$ of a test particle of mass 1 , given by

$$
\frac{1}{2} \bar{v}^{2}=\frac{1}{2} \int_{-\infty}^{+\infty} p(v) v^{2} d v
$$

where $\cdot-$ denotes the expectation with respect to the marginal distribution $p(v)$. In an environmental setting, within our toy model, we may think of this as the mean kinetic energy intensity of particle movements, making up e.g. storms, in an environment where (inverse) temperature is distributed according to a $\chi$-square distribution. For $q$-statistics one can explicitly calculate the above integral as

$$
\frac{1}{2} \bar{v}^{2}=T_{0} \frac{3-q}{5-3 q}
$$

where $T_{0}=1 / \beta_{0}$ is the mean temperature.

We now see that the effects of global warming are more dramatic if temperature fluctuations $(q>1)$ are taken into account: The change with global warming (i.e., a slight increase of $T_{0}$ ) is given by

$$
\frac{1}{2} \frac{d}{d T_{0}} \bar{v}^{2}=\frac{3-q}{5-3 q} .
$$

If $q=1$ (no temperature fluctuations), the quantity on the right hand side is just 1 , which corresponds to the mean field response to global warming if no temperature fluctuations are taken into account. However, as shown in this paper it is important to take into account the distribution of local temperature, which creates, according to eq. (5) or (9) an effective $q>1$. For $q>1$ the term on the right-hand side of eq. (12) is larger than 1, thus meaning a more dramatic response effect to global warming. The expected kinetic energy of a test particle grows stronger than in the case where there are no temperature fluctuations, and thus ultimately we also expect that storm intensity or other environmentally interesting observables will respond more strongly to tiny increases of $T_{0}$.

\section{CONCLUSION}

In this paper we have analyzed in detail the superstatistical distributions $f(\beta)$ relevant for a generalized statistical mechanics description of complex systems that are coupled to a changing temperature environment on planet earth. These local complex systems may include thermodynamic devices working outdoors, but in a more general setting can also include local ecosystems and other environmentally relevant subunits. We looked at local inverse temperature distributions for numerous examples of spatial locations. If seasonal periodicity is not removed from the data, typical distributions have a double-peak structure, with details of the two peaks (their intensity, variance, and intermediate connection) dependent on the local climate. The double-peak structure implies that conventional type of superstatistics [1] for single-peaked distributions is not applicable. If data are restricted to particular months, thus eliminating seasonal variations, one obtains single-peaked distributions, which in reasonably good approximation are Gaussian, at least in the vicinity of the maximum and for most examples of spatial locations we have chosen. However, for some locations (e.g. Vancouver, Darwin) we do see anomalous tails, in line with recent observations reported in [46], though in our case this seems to be more the exception than the rule. A planned future project is to analyse the tails in temperature distributions (as well as precipitation statistics) in more detail at more locations, taking into account known techniques from extreme event statistics [45].

On time scales of several decades our data clearly show the effect of global warming, with the rate of average increase in temperature depending very much on spatial location. Some examples of locations (Eureka, Dubai) were found where the rate of increase of average temperature is significantly higher than the global average. When looking at the response effects to global warming it is very important to take into account local temperature distributions and thus to proceed to a superstatistical description. We showed (for a simple toy model system) that the effect of global warming on kinetic energy expectations (of moving particles) is indeed stronger if temperature distributions at the various spatial locations (rather than constant temperature) are taken into account. 


\section{ACKNOWLEDGEMENT}

G.Cigdem Yalcin was supported by the Scientific Research Projects Coordination Unit of Istanbul University with project number 7441. She gratefully acknowledges the hospitality of Queen Mary University of London, School of Mathematical Sciences, where this work was carried out. Christian Beck's research is supported by the EPSRC grant 'Flood MEMORY'.

[1] C. Beck and E.G.D. Cohen, Physica A 322, 267 (2003)

[2] C. Beck, E.G.D. Cohen, and H.L. Swinney, Phys. Rev. E 72, 056133 (2005)

[3] H. Touchette and C. Beck, Phys. Rev. E 71, 016131 (2005)

[4] C. Tsallis and A.M.C. Souza, Phys. Rev. E 67, 026106 (2003)

[5] P. Jizba, H. Kleinert, Phys. Rev. E 78, 031122 (2008)

[6] P.-H. Chavanis, Physica A 359, 177 (2006)

[7] S.A. Frank and D.E. Smith, Entropy 12, 289 (2010)

[8] C. Anteneodo and S.M. Duarte Queiros, J. Stat. Mech. P10023 (2009)

[9] E. Van der Straeten and C. Beck, Phys. Rev. E 80, 036108 (2009)

[10] R. Hanel, S. Thurner, and M. Gell-Mann, PNAS 108, 6390 (2011)

[11] K. Briggs, C. Beck, Physica A 378, 498 (2007)

[12] C. Beck, Phys. Rev. Lett. 98, 064502 (2007)

[13] L. Leon Chen, C. Beck, Physica A 387, 3162 (2008)

[14] A.Y. Abul-Magd, G. Akemann, P. Vivo, J. Phys. A Math. Theor. 42, 175207 (2009)

[15] K.E. Daniels, C. Beck, and E. Bodenschatz, Physica D 193, 208 (2004)

[16] C. Beck, Physica A 331, 173 (2004)

[17] S. Rizzo and A. Rapisarda, AIP Conf. Proc. 742, 176 (2004)

[18] D.N. Sobyanin, Phys. Rev. E 24, 051128 (2011)

[19] P.D. Dixit, arXiv:1210.3015

[20] C. Tsallis, J. Stat. Phys. 52, 479 (1988)

[21] C. Tsallis, Introduction to Nonextensive Statistical Mechanics, Springer, 2009

[22] C. Beck, Phys. Rev. Lett. 87, 180601 (2001)

[23] V. Lucarini, T. Nanni, A. Speranza, Nuovo Cimento C 27, 285 (2004)

[24] M.R. Attolini, S. Cecccinni, M. Galli, Nuovo Cimento C 7, 245 (1984)

[25] K.Y. Vinnikov, A. Robock, N.C. Grody, A. Basist, Geophys. Res. Lett. 31, L06205 (2003)

[26] A. Porporato, G. Vico, P.A. Fay, Geophys. Res. Lett. 33, L15402 (2006)

[27] M.R. Jones, H.J. Fowler, C.G. Kilsby, S. Blenkinsop, Int. J. Climatology (2012), DOI: 10.1002/joc.3503

[28] Australian Government Bureau of Meteorology www.bom.gov.au

[29] National Oceanic and Atmospheric Administration, National Climatic Data Center www.ncdc.noaa.gov

[30] Dubai Meteorology Ofice www.dia.ae

[31] D.E. Parker, T.P. Legg, and C.K. Folland. A new daily Central England Temperature Series, 1772-1991. Int. J. Clim., Vol 12, 317-342 (1992)

[32] United Kingdom National Weather Service www.metoffice.gov.uk

[33] Enviroment Canada Weather Office www.weatheroffice.gc.ca

[34] Hong Kong Observatory, The Government of the Hong Kong Special Administrative Region www.hko.gov.hk

[35] W. Köppen, Meteorologische Zeitschrift, 20, 3, 351 (2011)

[36] M. Kottek, J. Grieser, C. Beck, B. Rudolf, F. Rubel, Meteorologische Zeitschrift, 15, 3, 259 (2006)

[37] M. C. Peel, B. L. Finlayson, T. A. McMahon, Hydrol. Earth Syst. Sci., 11, 1633 (2007)

[38] Trenberth et al., Ch. 3, Observations: Atmospheric Surface and Climate Change, Section 3.2.2.2: Urban Heat Islands and Land Use Effects, p. 244, in IPCC AR4 WG1 2007.

[39] Jansen et al., Ch. 6, Palaeoclimate, Section 6.6.1.1: What Do Reconstructions Based on Palaeoclimatic Proxies Show?, pp. 466-478, in IPCC AR4 WG1 2007.

[40] S.J. Hassol, Impacts of a warming Arctic: Arctic Climate Impact Assessment. Cambridge, UK: Cambridge University Press. February 2005. doi:10.2277/0521617782. ISBN 0-521-61778-2.

[41] R. Vautard, P. Yiou, M. Ghil, Physica D 58, 95 (1992)

[42] M. Ghil and R. Vautard, Nature 350, 324 (1991)

[43] M.R. Allen, P.L. Read, L.A. Smith, Nature 355, 686 (1992)

[44] G. Katul, A. Porporato, R. Oren, Annu. Rev. Ecol. Evol. Syst. 38, 767 (2007)

[45] M.S. Santhanam and H. Kantz, Phys. Rev. E 78, 051113 (2008)

[46] T.W. Ruff and J.D. Neelin, Geophys. Res. Lett. 39, L04704 (2012) 